\title{
TRANSPORT PROTOCOLS
}

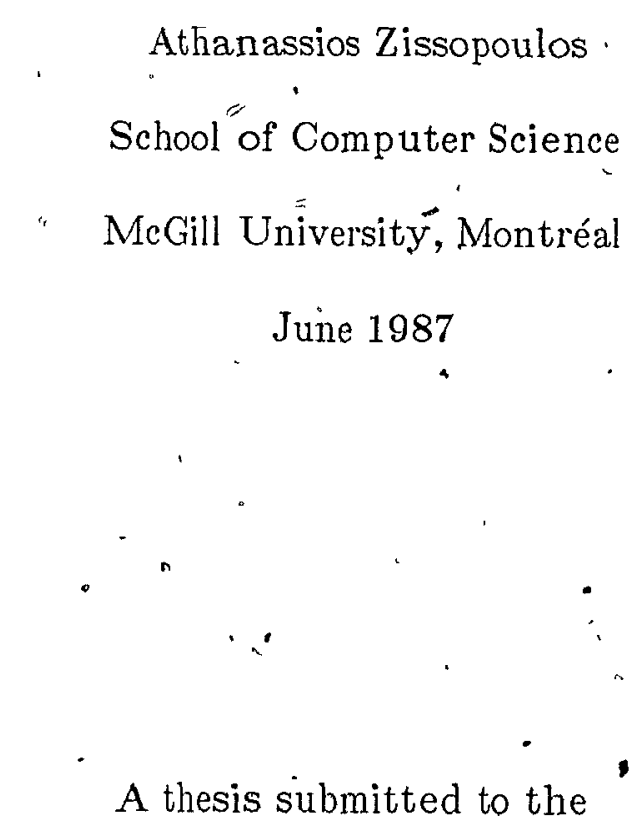

Faculty of Graduate \$tudies and Research in partial fulfillment of the requirements

- for the degree of Master of Science.

(C) A. Zissópoulos 


\section{$\cdots * \quad$ ACKNOWLEDGMENTS}

I would like to thank Prof. Carl Tropper, my thesis supervisò, for introducing me to the field (and for waiting while I picked daisies), for guiding me, and for being a friend.

'I am indebtied to my friends in heavy smokers' lab [EVEQ86]. Claude for the endless discussions and Lurcy for uncómplainingly inhaling the pollution we generated.

- ' ' '

I would also like to thank my parent\$ for their support and encouragement. Lastly, I would like to thank my wife Susan, who sat with me through interminable revisions, and without whose patience this thesis might have been finished érlier. 



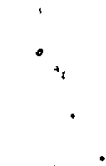

\section{- $\quad \therefore$ RESUME}

Nous présentons dans cette thè̀se un algorithme poứr répartir les mémoires tampons attribuées au niveaư transport d'un réseau à commutation de paquets. L'algorithme alloue les mémoires tampons jen fonction des longueurs estiméés des files d'attente des différentes catégories de trafic, assurañ ainsi une répartition équitable. E Én outre, une priorité plus élevée est dónnée aux catégories de trafic ayant les messages les plus coúrts. Ces catégories de trafic pourraient correspon. dre 'au trafic interactif' ainsi- qu'un trafíc dû au transfert de fichicrs. Un 'mécanisme évitant le verrou mortel 'dû aux réassemblages des paquets est incorporé dans l'algorithme. 


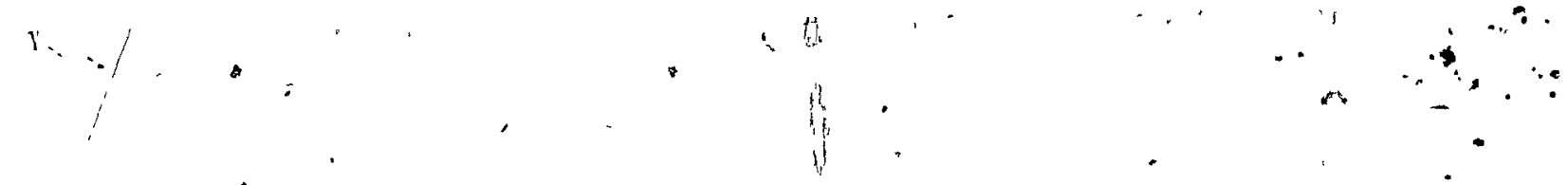

\section{ACKNOWLEDGMENTS \\ ABSTRACT}

.RESUME

\section{$\therefore$,}

Chapter 1

Introduction

Chapter 2

Transport Protocol

2.1 OSI Transport Protocol (TP)

\subsubsection{TP4}
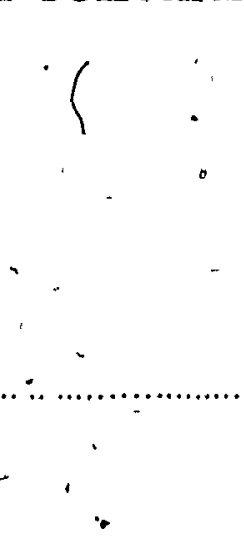

\section{Chapter 3}

\subsection{Transmission Control Protocol (TCP)}

Theoretical Underpinnings

3.1 Markov Decision Process (MDP) and Policy-Iteratıon Methọd

3.2 Time Series Analysis

Chapter 4

Previous Work

Chapter 5.

Buffer Allocation Algorithm

5.1 Observations

5.2 The Algorithm

\section{Chapter $\theta$}

Simulations

6.1 Network Model

62 Simulation Model

$\therefore \ldots$

Chapter.7

Results, Conclusion, Suggestions

7.1 Results ........

7.2 Summary and Conclusion

7.3 Suggestions for Future Work 


\section{Chapter 1 \\ ' Introduction '}

We present in this thesis a buffer, allocation algorithm which can be used in a transport protōol such as the OSI's 'TP class 2,4 protocols or the'ARPANET's TCP , The algorithm can be used in conjunction with the buffer reservation mechanism between a source-destination pair of hosts. The algorithm, if extended to account for different network categories'and different communication media, can also'be adopted for use at a gateway. Before we proceed, we give a brief' introduction to the terms found throughout the thesis.

A computer network is a collection of independent computers (hosts) which are able to communicate with each-other. The hosts are connected to a communication subnet which consists of switches (imps) connected to each other via some transmission" media (telephone, cable, satellite, and fiber optic.links). The rules that govern the communica- tion between the computers are called protocols. Several benefits arising from this interconnection are:

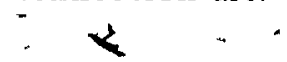

- the ability to use remote computers, remote programs and databases

- an increase in reliability due to the availability of alternative resources [TANE 8 1]

- electronic mail śrvices

- Typical services provided by a computer network are mail, news, and remote login.6 Future applications can, ínclude computer-aided education, teleconferencing and electronic funds transfer [TANE81]. There are two categories of networks, the local area and the wide area. In local area networks, which are usually owned py private organizations, the resources lie within a radius of a few kilometers and have high data rate 


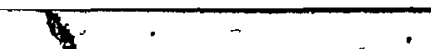

- 2 -

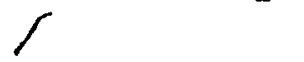

communication links (Husually coaxial cable or fiber optics) of the order of a few Mbps. In wide area networks, the resources are located fa $\overline{\bar{r}}$ apart (spread across a continent or around the world). The communication links, are slower (of the order of $50 \mathrm{Kbps}$ ) and may be owned by pujplic or private organizations Notworks can also be interconnected through a gateway, thus forming supernetworks. The role of the gateway is the transfer of messages/packets from one, network to another.

The first experimental (one node) network in North America was brought to life in 1969 [KLEI76] This arose from the ARPA community's realizing that it could take advantage of already existing but diversely located machines by interconnecting them.

- The interconnection was meant to increase the utrlization and the availability of the resources in an economical fashion. The efforts ultimately resulted in the ARPANET . 'packet switching network. During the two decades that, have since elapsed, a clearer understanding of networking and a more systematic design process has emerged Significant efforts by international organizations such as the International Standards Organization (ISO) and the Comité Consultatif Internationale de Télégraphique et Téléphonique (CCITT) are leading towards a standardization of the protocols Typical. examples of. today's successfully operating networks are.DATAPAC (Canada), TYMNET (USA), TRANSPAC (France), SNA (IBM), EIN (Europe), EUNET (Europe), and CSNET (USA) [QUAR86], [SCHW87].

- One of the first efforts, with the ultimate goal being the international standardization of protocols, is the reference model for Open Systems Interconnection (OSI) suggested by the ISO [ZIMM80]. It consists of seven layers, all of which are derived from similar theoretical backgrounds. Each layer uses the services provided by the layer below and provides services to the layers above., The three lower layers (the Physical; Data link and Network) deal with the communication network, while the upper four 


\section{$-3-$}

layers (the Transport, the Session, the Presentation and the Application) deal with the end users.

The Transport layer, which we deal with, guarantees reliable and efficient end-toend communication. Efficiency can be achreved by multıplexing and segmertıng, while reliability is achieved by reassembly and, reotedering, error detection and recovery techniques, and flow control. These techniques are implemented through a program called the transport station.

- Flow control is a set of rules needed to regulate the traffic between two processes, In this, way preventing buffer overflow at the receiver. It occurs in the lower layers as well as in the upper ones. One common technique of flow control is the sliding window mechanism. With this technique, the maximum number of unacknowledged messages between a pair of processes is restructed to the value of the window (a window of one is the stop-and-wait mechanism) The waty it works is as follows. each acknowledgment carries back two numbers One is the sequence number of the next expected messàge and the other is the credit number (that is, the number of messages permitted to be sent until the next allocation). The sequence number is a unique number which is assigned to any data unit transferred between a source-destination pair It'serves to identify the unit during the transmission phase, and helps reordering during the reassembly of a completely received message Ali in teresting survey on flow control can be found in GERL80

TP2 and TP4, ISO transport protocol international standands, and the ARPANET's TCP use a sliding window of sequence numbers Credits are allocated by an exchange of ACK TPDUs or ACK segments. Initial allocation occus during the connection establishment phase In TCP, the credits are allocated in bytes while in TP2 or TP 4 they are designated in transport protocol da, a unts (TPDUs) TPDUs can valy in size from 128 to 8192 octets (bytes) A detalled description of the TP and TCP.credit alloca- 


\section{$-4-$}

tion mechanisms can be found in SCHW87 or STAL87. For even more detail the specifications, ISO84a, NBS83a, and TCP83 may also be consulted.

The buffer allocation a gorithm whìch we developed allocates buffers as a function of the message length and the estimated queue length at the destmation transport station Space allocated for a message category is accessible to that category and to all the categorıes hàvıng smaller message lengths, ie, small messages have a higher prouty than the larger messages Categon as ale determined by the length of the message and are user defined The objective is to provide a small delay for single packet traffic (Interactive or ${ }^{\circ}$ mail) and at the same time ensure acceptable throughput levels for longer messages (file transfer, videotext).

The algorithm is comprised of two parts In the first part, the expected buffer occupancy for different categories of messages is estimated In the second part, each category receives its predıcted space, whlle free space is made avallable to every other category Furthermore, a reassembly deadlock avoidance mechamsm is incorporated withn the algorithm for reasons explained below.

When the destination host buffers data and delivers them only upon reception of the complete message, a reassembly deadlock may arise. This event occurs whign buffers are fully occupied by partially recerved messages. Since no more message segments can be sent, obviously none can be received Hence; a deadlock situation, which necessitates the employment of our reassembly deadlock avoldance algorithm

In order to examine the peiformance of the algorithm, we have undertaken smulations comparing the proposed credit allocation to the one employed by the TP ur TCP The simulations were carıled out using the PAWS (Peiformance Analyst's Workbench System) s!mulation tool [PAWS84] 
The remainder of the thesis is organized as follows Chapter 2 gives a description of the most popular transport protocols, the TP 0 to 4 class and the TCP Chapter 3 deals

- with the theoretical underpinnings for our approach to the problem as well as that of the previous work Chapter 4 gives a rather extensive discussion of the plevious work done in the area. In Chapter 5, we discuss the rationale for our using the forecasting $\because$ approach, describe the buffer allocation algorithm in more detail, and present the deadlock avoldance algorithm. Chapter 6 'describes the simulation model and the simu- lation tools Finally, Chapter 7 contains the results, conclusions, and suggestions for

$\therefore \quad$ future work. $\quad$ :.

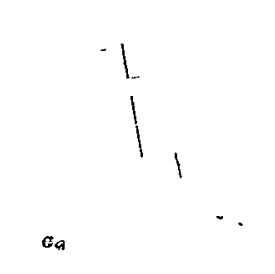




\section{Chapter 2 Transport Protocol}

The transport layer is the layer that is responsible for transferring data between two user processes (the layers above) It is the first layer, starting from the bottom in the ISO herarchy, that deals with end-to-end communication. This is in contrast to the lower layers, which are concerned with communication between (network) switches. The tasks of this layel include naming and addressing of user processes, connection establishment and termination, multiplexing and splitting of connections, segmenting and reassembly of messages, error recovery, and flow control The transport layer guarantees a quality of service to the users that is independent of the service provided by the underlying layers and network(s) The program which implements these services is called the transport station It runs on a host computer and is (or it could be) part of the' host's operating system.

Services are requested by name from the user, hence it is the responsibility of the transport layer to map the name to its transport address: These addresses can be either herarchical or flat [TANE81]. The hierarchical address is a set of disjoint fields numbered similarly to telephone numbers. Knowledge of the address determines the exact location A flat address is just a unique number with no relationship to the rest of the addresses.

Once the address is known, the connection establishment.phase proceeds. Part of this phase is the negotiation of the quality of service (acceptanceof the suggested quality of service by the sender or modification by the receiving user) between the user processes The quality of service deals with parameters such as sequence number space, 
data unit size, throughput and delay requirements, etc. This phase's being successful leads to the data transfer phase.

If high throughput is the issue, the transport connection may be split in to multiple network connections. On the other hand, the multiplexing of multiple transport connections onto a single network connection can reduce the cost of the connection. Segmenting may occur both if the transport data unit exceeds the network data unit or if the destination reduces the proposed data unit size. Segmenting at one end eauses data reassembly (reordering of the data) at the other end, since data have to be delivered to the user as they were sent Flow control prevents overflow of the destination's buffers by a fast source Transmitted (unacknowledged) data can be buffered at the sender, at the desti"nation, or both, depending upon the relability of the underlyng network(s). At the destination, buffer späce is allocated, If it is avallable, upon request The laçk of buffer space naturally causes the sender to walt.

- The transport protócol should be able to detect, and recover from, any' detrimental Situations such as duplicate data removal, reassembly deadlock, etc

In the following, we describe the ISO TP 0-4 class transport protocol, with emphasis on TP4, and the ARPANET's TCP.

\subsection{OSI Transport Protocol (TP)}

$\mathrm{TP}$ is the ISO international standard transportalayer protócol [ISO84a], [NBS83a]. . In an attempt to deal with different categories of network services, the protocol consists of five distinct classes. Going from class 0 to class 4 , the protocol provides increasingly morc complex functions. The choice is determined by the services offered by the underlying network connections 
There are three,types of network connections [SCHW87]. These network connec$\because$ tions are assumed to be less reliable as one moves from Type-A to Type-C. In a Type-A connection, there is no out of órder arrival or loss of packets (ie. networks with virtual circuit services). The rate of both the errors and the signalled failures to the transport

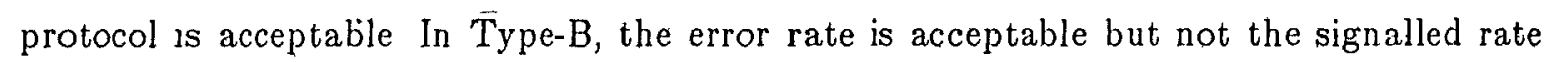
of falures. In this case, the transport protocol should provide error recovery Finally, in Type-C, the error rate is also not acceptable and the transport protocol should support functions for error detection and recovery (1e. networks with datagram services) Classes 0 and 2 operate on Type-A network connections, classes 1 and 3 on Type-B and class 4 on Type-C. Class 0 provides set up connection, segmenting, ánd data transfer services. Class : providés sımple error recovery services as, well Class 2 provides multiplexıng, class 3 provides error recovery and multiplexıng, and finally class 4 provides error detection and recovery

\subsubsection{TP4}

As was mentroned above, TP4 was designed to work under pathological situations

"The basic unt of information exchange between the transport stations is referred to as the Transport Protocol Data Unit (TPDU).

A connection is established between two transport addresses, the transport service access point idéntifiers, by a Connection Request (CR) TPDUं (maximum size for each TPDU header is 254 octets). In the case that both ends try to establish a connection, then two connections are set, up. The CR TPDU, among its other duties, suggests the data TPDU size (possible range between 128 octets to 8192 octets), the sequence number spacé ( 7 or 31 bit field), the initial credit allocation ( 4 or 16 bit field) for traffic 
flowing from the receiver to the sender, and the quality of service parameters The receiver responds either with a Connection Confirm (CC) TPDU accompanied by an initial credit allocation, in case it wishes to communicate, or with a Disconnect Request (DR) TPDU in case it doesn't Moreover, it is permitted to decrease (although not beyond a specified limit) any parameters (suggested by the sender), but is never allowed. to increase them $\mathrm{CR}$ and $\mathrm{CC}$ can carry up to 32 octets of data, while a $\mathrm{DR}$ may carry up to 64 octets of data

Upon completion of the connection establishment phase, which uses a three-way handshake (CR, CC an'd ACK or DT), the protocol enters the data transfer phase by transmitting Data (DT) TPDUs. The DT TPDUs are acknowledged by Acknowledgment (ACK) TPDUs, the delay of which causes data to be retransmitted after the expiration of the appropriate tımer The destination transport station can deliver the data to the transport user either piece by piece or after receipt of the complete message The choice is implémentation dependent.

Flow control is carried out by a sliding window mechanısm. More' precisely, each ACK TPDU carries the sequence number of the next expected TPDU (lower edge win-

- dow) plus the number of credits allocated (the number of DT TPDUs the sender is permitted to send until the next allocation).

Termination of the connection is carried out by a Disconnect Request and Disconnect Confirm (DC) TPDUs. Any data that were sent before the termunation and were not delivered after the receipt of the DR and DC TPDUs, are, discarded. The Graceful close Request (GR) TPDU helps these waiting data to be delivered (avallable only in the NBS FIPS version). It is acknowledged by an ACK TPDุU

Connectionless data transmission is accomplished by using a single Unit Data (UD) TPDU. 
Fast delivery of urgent data $(\leq 16$ octets) can be done with the Expedited Data (ED) TPDU, which'should in turn be acknowledged by an Expedited Acknowledgment (EA) TPDUं Only one ED TPDU can be outstanding at any time.

Finally, transmission error detection is accomplished by using the Fletcher check-a summing procedure. Also, since ęach ${ }^{-}$TPDU is always acknowledged by another TPDU (CR is acknowledged by a CC, CC by DT or ACK, DT by ACK, and ED by EA), further error detection arid ręcovery is accomplished by the acknowledgments and the timers which are set during transmission.

\subsection{Transmission Control Protocol (TCP)}

- TCP was designed to work over uñreliable networks [TCP83]. It operates in tandem with the Internet Protocol (IP), whose purpose is to support the interconnection of networks The TCP transport station accepts messages of arbitrary length, the letters, and splits them into segments $(\leq 65 \mathrm{~K})$, which are the basic data units There are four - types of segments DATA, SYN, FIN and ACK. The minimum size of the header is 20 bytes.

The connection is established by, the SYN (from both sides) and ACK segments, and a three-way handshake is employed. There is only one connection set up between each pair of source-destination addresses (the sockets as they called in TCP). This is an effect of the fact that the sending process can be in either active or passive mode When in active mode, the sending process denotes a destination with which it would like to connect, whereas in passive mode it waits for connection establishment by another process. Data are carried on DATA segments and are acknowledged by ACK segments. ACK segments, in turn, can be piggybacked onto DATA segments. They may ack- 
nowledge segments individually or in groups. Timers are set up upon the transmission of DATA segments, and their expiration causes DATA retransmission.

Flow control is' carried out by employing a variable-size sliding window (16 bit field), but the protocol allocates bytes, in contrast to TP4 which allocates ${ }^{-}$TPDUs (blocks of data). The protocol encourages the sender TCP to transmit periodically, even if the window is zero, in order to avoid the possibllity of a deadlock in case ACK segments were lost. Since each byte of data has its own sequence number, the sequence number space is extremely large (32 bit field) This guarantees that old duplicates have vanished before the same sequence numbers are used again (ie "wrap-around") In addrtion, each segment has a maximum lifetime in the network, after which it is destroyed. -

A connection is terminted by the issuance of an FIN segment accompanied by an ACK segment from the other side A Graceful close mechanism is also avallable, and requires FIN segments by both sides followed by an ACK' segment (three-way handshake termination)

Data marked as URGENT are transmitted immediately by the TCP. There is no limit to the number of outstanding data of this kind A PUSH service can also be initiated by the sender process in order to receive fast response by the destmation process. This "service is meant to help "the interactive users and to avoid the possibility of a deadlock.

Finally, checksumming procedures employ the one's complement algprithm, while error detection and recovery is incorporated in to the protocol

- We close this section by pointing out. that certain portions of TCP have been adopted, although with few modifications, by TP4. These include the three-way handshake connection estabfshment and the flow control mechanısm. 


\section{Chapter 3}

\section{Theoretical Underpinnings}

In this chapter, we give a brief introduction to the mathematical theory involved both in the previous work relating to the problem of buffer allocation and flow control and in our own approach to the problem. The theories introduced are the Markovian Decision Processes and Time Series Analysis. A more complete analysis may be found in [HOWA60] and [HEYM84] for the Markovian Decision Processes and in [BOX76] for Time Serits Analysis, from whence this description was drawn.

\subsection{Markov Đecision Procèss (MDP) and Policy-Iteration Method $"$}

"A Discrete Time Markov Chain is a set of random variables $X_{1}, X_{2}, . ., X_{n}$, which satisfies the following equality

$$
P\left[X_{n}=\jmath \mid X_{1}=\imath_{1}, X_{2}=\imath_{2}, . ., X_{n-1}=i_{n-1}\right]=P\left[X_{n}=j \mid X_{n-1}=\imath_{n-1}\right]
$$

that is, the probability that the process is moving to the next state is dependent only on the current state (or in other words the history of the process is summarized in the current state) Such a process is described completely by the set of states $S$ and a transition (stochastic) matrix $P\left[p_{i j}\right]$ where $p_{i j}=P_{-}\left[X_{n}^{\prime}=j \mid X_{n-1}=i\right]$

The probability of finding the system in state $j$ after $n$ transitions is defined by $\pi_{j}(n)=\sum_{i=1}^{|S|} \pi_{i}(n-1) p_{z}$, or in vector form by $\pi(n)=\pi(0) P^{n}$. When this state occupancy vector is independent of the initial state for a large number of transitions $n^{\circ}$ (an ergodic process), the vector is called a limiting state probability vector. This quantity 
is of interest since it describes the process sufficiently The transient behavior of the system can be found by applying $z$-transform analysis. It can be showm that $\Pi(z)=\pi(0)[I-z P]^{-1}$ where $\Pi(z)$ is the $z$-transform of the $\pi(n)$ vector and $I$ is the adentity matrix. The inverse transform of the matrix $[I-z P]^{-1}$ consists of two parts; the steady state portion, $S$, and the tr nsient behavior portion, $T(n)$. The $(i, j)$ element of the matrix gives the probability that the system will occupy state $J$ after $n$ transitions given that state $i$ was the initial state The transient part (which is a function of $n$ ) decreases to zero as $n$ increases Each row of the steady state matrix, $S$, is the limiting state probability vector of the process.

A Markovian Décision Process (MDP) is the model obtained from a Markov chain by adding actions and rewards An action refers to the feasible paths (alternatives) taken in every state and Is defined hoach state An immediate reward (which is a random variable) refers to the positive or negatıve gain from taking a possible action. whlle being in a particular state The next state and the immediate reward are independent of the history, given the current state and action (the Markovian assumption). The choice of an action can affect the future in terms of the information available, the feasibility of an action, and the gain of the rewards. .

A quantity of in terest, the exfected total reward $u_{?}(n)$ after $n$ transitions given that the system is currently in the state $i$, is given by

$$
u_{i}(n)=q_{i}+\sum_{j=1}^{|S|} p_{i j} u_{j}(n-1) i=1,2, \ldots|S| n=1,2, \ldots
$$

V I that is, the sum of the expected immediate reward due to transition out of state $i$ and -

- the expected reward from state $j$ with $n-1$ transitions. The above equation in vector

: $\quad$ form is $u(n)=q+P u(n-1), \quad n=1,2,3, \ldots$ 
A $\bar{z}$-transform analysis shows that $u_{i}(n)=n g_{i}+u_{i}^{\prime}, i=1,2,3, \ldots,|S|$, where $g_{i}$ is the gain of the state $i$ and $u_{i}$ is the intercept of the asymptote $u_{i}(n)$ at $\bar{n}_{\bar{i}}=0$. In the case that the Markovian Decision Process is ergodic, then $g_{1}$ is the same for all $i$, $\checkmark$ : and is called the gain $(g)$ of the process.

If we introduce the notion of an alternative action, then by applying the Principle 4. of Optimality, the formula for the expected total reward becomes

$$
u_{i}(n)=\max _{k}\left\{q_{i}^{k}+\sum_{j=1}^{|S|} p_{\eta j}^{k} u,(n-1)\right\} \quad i=1,2, \ldots,\left|S^{\prime}\right| \quad n^{\prime}=1,2,3, \ldots
$$

which states that having found which actions to employ up to transition $n-1$, the above equation is maximized by finding which action maximizes $q_{i}{ }^{k}$. The action selected for each state is called the decision for this state and the set of decisions (vęctor) for all states is called the policy. The optımal policy is the policy that maximizes the expected total reward.

One of the (efficient) methods of finding the-optimal policy is the Policy-Iteration method. This method consists of an iteration cycle which in turn consists of two parts a) the value-determination part and b) the policy-improvement part.

In the first part, the relative values $u_{i}$ (asymptotic intercepts of $u_{i}(n)$ ) and the gain $\dot{g}$ for a given policy are calculated by solving a system of $|\vec{S}|$ linear equations. This system is produced by substituting the set of equations $u_{i}(n)=n g+u_{i}$ into the set $\ddot{u}_{i}(n)=q_{i}+\sum_{j=1}^{|S|} p_{i j} u_{j}(n-1)$. The result is the set of $|S|$ equations

$$
g+u_{i} \cdot=q_{i}+\sum_{j=1}^{|S|} p_{i j} u_{j} \quad i=1,2, \ldots,|S| \quad n=1,2, \ldots
$$

with $|S|+1$ unknowns. This system is solved by setting one of the $u_{i}$ equal to zero. The values found are used in the next step of the iteration cycle. 
In the policy-improvement part for each state, the action $k$ that maximizes the quantity $q_{i}^{k}+\sum_{j=1}^{|S|} p_{i j}^{k} u_{j}$ is found. The action $k$ becomes the new decision, $q_{s}{ }^{k}$ becomes $q_{s}$ and $p_{i j}^{k}$ becomes $p_{i}$, and the iteration cycle is repeated until convergence of whe algorithm is met Each step of the iteration produces a higher gain than the previous one. The cycle terminates, when two successive policies are identical. It can be proven that at this point the optimal policy is found.

\subsection{Time Series Analysis}

Time serıes analysis is, an analysis of dependent time-sequenced observations $z_{1}, z_{2}, ., z_{t}, \ldots$ (time series). The objective of the analysis is the derivation of a model which can give a) useful information about the system which gen'erates the series, and b) optimal predicted future valựes of the series. This analysis, then, can be thought of as an extrapolation (projection) of past values in to the future

The knowledge of past behavior can be used by many models One family of models which guarantees optimal forecasting (in terms of smaller square forecast error than any other single series) is the $A R I M A$ model, which is an Integration of the Auto-Regressive $(A R)$ and Moving Average $(M A)$ model. We proceed to some definitıons which may ease the description of the $A R I M A$ models, as well as the procedures for adopting each model.

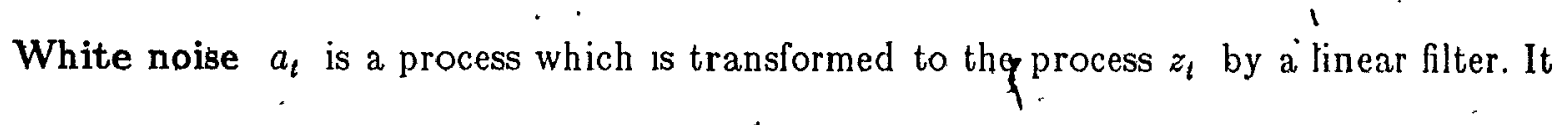
is assumed to be normally distributed.

The deviation from the estimated mean $\bar{z}$ is defined as $\tilde{z}_{t}=z_{l}-\bar{z}$

A backward shift operator is defined as $B^{n} z_{\ell}=z_{l-n}$

The first difference is defined as $w_{t}=z_{t}-z_{t-1}=(1-B) z_{\imath}$. The $\mathrm{n}$ th difference is 
defined as $(1-B)^{n} z_{t}$. Differencing is employed when the mean of the series is not stationary.

The autocorrelation function is a function that measures the correlation between ordered pairs $\left(\tilde{z}_{t}, \tilde{z}_{t+l}\right)$ drawn from a time series.

The partial autocorrelation function is a function that measures the correlation between ordered pairs $\left(\tilde{z}_{t}, \tilde{z}_{t+l}\right)$ drawn' from a time serıes, also taking in to account the effects of the observations lyng between these ordered pairs.

An $\operatorname{AR}(p)$ model of order $p$ is

$$
\tilde{z}_{t}=\phi_{1} \tilde{z}_{t-1}+\phi_{2} \tilde{z}_{t-2}+\cdots+\phi_{p} \tilde{z}_{t-p}+a_{t}
$$

or

$$
\left(1-\phi_{1} B-\phi_{2} B^{2}-\cdot \cdot-\phi_{p} B^{p}\right) \tilde{z}_{t}=a_{t}
$$

In this model, the present $t_{i}$,value of the process is defined as a linear aggregation of previous values and of white noise.

The MA(q) of order $q$ model is defined as a linear aggregation of previous white noise values, $a_{t}$

$$
\tilde{z}_{q}=a_{t}-\theta_{1} a_{t-1}-\theta_{2} \dot{a}_{t-2}-\cdots-\theta_{q} a_{t-q}=\left(1-\theta_{1} B-\theta_{2} B^{2}-\cdots-\theta_{q} B^{q}\right) a_{t}
$$

Finally the $\operatorname{ARIMA}(\mathbf{p}, \mathbf{d}, \mathbf{q})$ model is defined as an integration (differencing) of the above models

$$
\left(1-\phi_{1} B-\phi_{2} B^{2}-\cdots-\dot{\phi_{p}} B^{p}\right)(1-B)^{d} z_{t}=\left(1-\theta_{1} B-\theta_{2} B^{2}-\cdots-\theta_{q} B^{q}\right) a_{t}
$$

Special cases of the $\operatorname{ARIMA}(p, d, q)$ models are the $\operatorname{ARIMA}(0,1,1)$ or $z_{t}=z_{t-1}-\theta a_{t-1}+a_{t}$ which is called Exponentially Weighted Moving Average (EWMA), the $\operatorname{ARIMA}(1,0,0)$ or $\tilde{z}_{t}=\phi_{1} \tilde{z}_{t-1}+a_{t}$ which is a Markov Process and the $\operatorname{ARIMA}(0,1,0)$ or $z_{i}=z_{t-1}+a_{t}$ which is a Random Walk , 
An iterative procedure that leads to the selection of the appropriate model out of 'the $\operatorname{ARIMA}(p, d, q)$ family models has been deyeloped by Box and Jenkins [BOX76]. The suggested sample size is comprised of approximately fifty observations. The procedure applies only to time series that have a mean, varıance and an autocorrelation function invariant through time (stationary series) Nonstationary series can be transformed to stationary ones, often by differencing. The iterative procedure has three phases During the first phase, identification, the statistical relationships between the roughly estımated and the theoretical values of both the autocorrelation and the partial autocorregtion functions are checked, and the model with the higher colrelation is chosen. In the estimation phase, a mole efficient estimation of the coefficients of the model chosen during the previous phase is attempted 'If these estimates do not satısfy certan condıtıons for, , stationarity; invertibllty, etc, the model is rejected Finally, during the last phase, diagnostic checking, the statistical adequacy (ie. analysis of the estimated random shocks) of the ,model is checked Fallure to, meet these tests causes rejection of the model and repetitigh of the procedure. Otherwise, the mode is used for forecasting.

- Having found the appropriate model and its coefficients, the future values of the series at lead time $l \geq 1$ can be forecasted given the knowledge of the series up to some origin time $t$ The forecast of $\hat{z}_{t+l}$ is equal to $E\left(z_{t+l} \mid z_{t}, z_{t-1}, ..\right)$. The conditional expectations are calculated by replacing actual values for the past observations $\dot{z}$ and random shocks $a$, and forecasting $\tilde{z}$ for future values. Future random sliocks are approximated by zeroes.

Application of the above into an EWMA givos $\hat{z}_{l}(l)=(1-0) z_{l-1}+0 \dot{z}_{l}-1(l)$. The above formula shows that small values of $\theta$ place more emphasis on the new observations than on the ones from the past. Large values of $\theta$, on the other hand, give more emphasis to a weighted average of the past history. A fascinatung thing about the 

To date, there have been few efforts in the modelling of the buffer (credit) allocation in transport level protocols. We discuss three papers here which deal with the modelling of an end-to-end window flow control mechanism. This mechanism is quite similar to the one employed by TP $\dot{4}$ and TCP.

The first paper which we discuss, KLEI80, develops a queueing model with a window flow control policy for end-to-end protocols. The queueing model employed does not, provide for a closed form solution, so numerical techniques were adopted. The parameters of the system are invariant despite fluctuations in the system load. The picture below depicts the structure of the model.

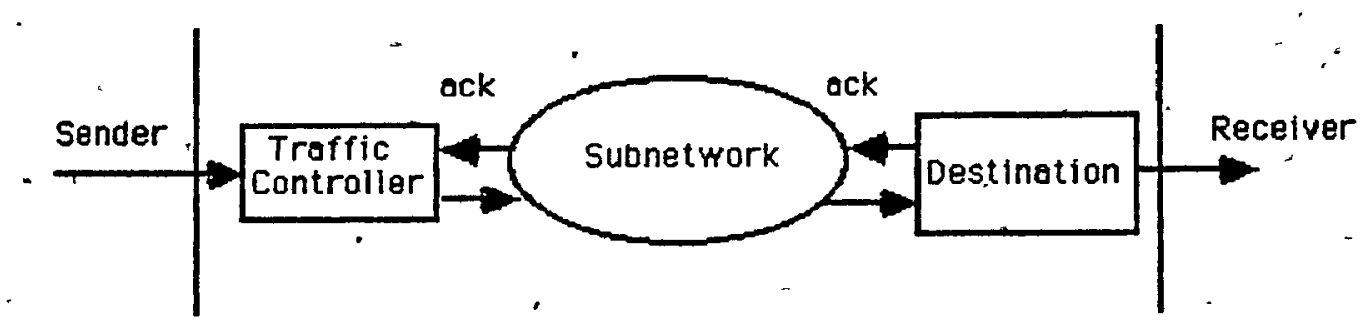

fig. 4.1

The task of the traffic controller is to kep the number of outstanding messages between the source and the destination node below a certain value $(w)$. More specifically, before being accepted in to the network, each message grabs a token which it keeps until the receipt of an acknowledgement In the case where an acknowledgment doesn't arrive before the traffic controller's specified timeout value $\tau$, another copy of the message is sent to the network Only the last acknowledgment is accepted by the traffic 
controller.

The destination node is modelled as a finite capacity queue. Lack of buffer space causes messages to be rejected without acknowledgment Duphcates are removed and while only the first copy of the message is passed to the receiver's host, acknowledgements are issued for all of them

- The round trip subnetwork delay is represented by an Erlang distribution Only singlèn packet messages are considęred, and the model operates under a heavy traffic assumption, that is, each time the traffic controller is ready to accept a new message, the sender has one ready The model focuses on a single connection

The following assumptions were made.

a) exponential message length distribution with mean $1 / \mu$

b) Poisson arrival rate with mean $\lambda$

c. independence assumption

d) the acknowledgments are i 1.d drawn from Erlangian distribution of degree tivo

e) finite buffer space at the destination

f) negligible transmission errors

g) the arrival rate at the destination after the removal of the duplicates it is assumed to be Polsson.

A brief discussion of the analysis is given below:

The acknowledgment delay distribution is given by $F_{\hat{l}_{a}}=\left(1-P_{l}\right)\left[1-e^{-\Lambda t}-\Lambda t e^{-\Lambda t}\right]$ where $=\gamma_{1}-\lambda_{e}$, (assumption $\mathrm{d}$ ), $\lambda_{e}$ is the effective network traffic rate, $\gamma_{1}=\mu C_{1}$, and $C_{1}$ is the capacity of the channels. $P_{l}$ is the probability that an acknowledgment is not recelved.

The retransmission probability $P_{r}$ is given by 


$$
\because \quad P_{r}=P_{1}+\left(1-P_{l}\right) \operatorname{Prob}\left[\hat{t}_{a}>\tau \mid \text { no loss }\right]=P_{l}+\left(1-P_{1}\right)(1+\Lambda \tau) e^{-\Lambda r}
$$

The effective input to the network, then, is given by $\lambda_{e}{ }^{\circ}=\frac{\lambda}{1-P_{r}}$

The time a message occupies a buffer at the traffic controller is .

$$
T_{o c}=E\left[\hat{t}_{a} \cdot \mid \hat{t}_{a} \leq \tau\right]+\frac{P_{r}}{1+P_{r}} \tau=\frac{2}{\Lambda}+\frac{P_{1}+\left(1-P_{1}\right) e^{-\Lambda} t^{\prime}}{\left(1-P_{1}\right)\left(1-[1+\lambda \tau] e^{-\Lambda t}\right)}
$$

The maximum input rate is related to $w$ and $T_{\text {or }}$ lyy $\lambda^{*}=\frac{w}{T_{o c}^{*}}$, where $T_{\text {or }}^{*}$ is the buffer occupancy time when the input rate is maximum $\lambda_{e}$ now is

$$
\begin{aligned}
& \lambda_{\dot{e}}^{*}=\frac{w}{\left(1-P_{r}\right) T_{o c}^{*}}=\frac{w 1}{\left.2\left(1-P_{l}\right)(1-\mid 1+\Lambda \tau) e^{-\Lambda !}\right)+\left|P_{l}+\left(1-P_{l}\right) r^{-\Lambda 1}\right| .1 ;} \\
& \quad=G_{1}\left(\tau, w, \gamma_{1}, P_{l}, \lambda_{e}\right)
\end{aligned}
$$

The unknowns in the equation are $\lambda_{t}$ and $P_{l}$ At cqullbrum, the mput ate fo the network is equal to the output rate of messages fiom at The sumbal ante to fhe destmatum buffer is $\lambda_{d}=\frac{\lambda^{*}}{1-P_{l}}$. The destmation buffer is modelled an an $M / M / 1 / 13 /$ quene (H beng the numbers of buffers at the destmation), so

$$
P_{1}=\frac{\gamma_{2}^{-}-\lambda_{d}^{*}}{\gamma_{2}^{B+1}-\lambda_{d}{ }^{H+1}} \lambda_{i} B=\left(i_{2}\left(\gamma_{2}, B, I_{1}, \lambda_{r}\right)\right.
$$

where $\gamma_{2}=\mu C_{2}$ and $C_{2}$ s the sulput channel capactly

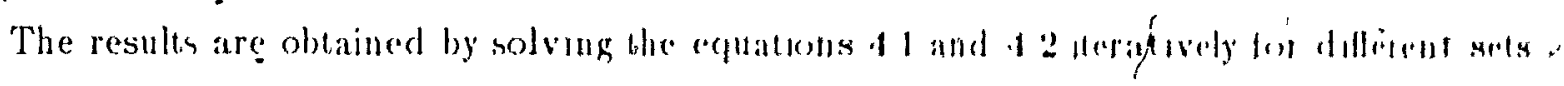

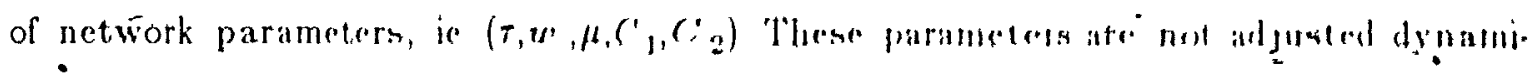
cally and fience, we have a model of stifie flow crontrol

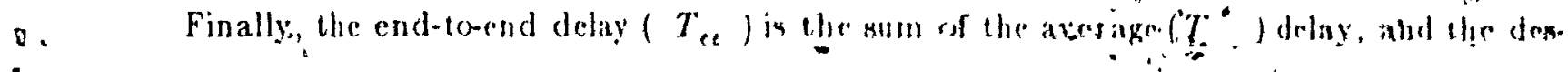
tination buffer delay $\left(T_{d w}\right)^{\circ} \ldots$ : 
h

$$
T^{*}=\frac{1}{\gamma_{1}-\lambda_{e}^{*}}+\frac{P_{l}}{1-P_{l}} \tau
$$

and

$$
T_{d s t}^{*}=\frac{\left(1 / \mu C_{2}\right)}{1-\rho_{d}^{*}} \frac{1-(1+B) \rho_{d}^{* B}+B \rho_{d}^{* B+1}}{1-\rho_{d}^{* B}}
$$

where $\rho_{d}^{*}=\lambda_{d}^{*} / \mu C_{2}$ is the utalization at the destination

The analysis demōnstrates that.

1) for a fixed $w$, throughput increases with timeout $\tau$ until $\tau^{\prime}$ reaches a certain value, whereupon throughput begins to decrease

2) for fixed values of $\tau$, throughput increases as $w$ increases, but after a certan value of $\bar{w}$ it starts to, decrease and,

3) for a fixed end-to-end delay, throughput increases with buffer capacity

Also, the analysis indicates that for a fixed value of $w$ there is a value of $\tau$ that maximizes throughput

In the second paper we discuss, KERM80, the authors use the ressalts of the previous paper on static flow control and develop an algorithm in which the window is computed dynamically As pointed out by the authors, the key idea behind this model is to prevent input to the network when the destination buffer is full This in turn prevents future retransmissions and rejectıons of messages, and as a consequence, unnecessary loading of the network The mathematical model developed is based on Markovian Decision theory. Since the exact solution grows exponentially, the authors develop a heuristic solution. This model also focuses on a single connection

The structure of the model is basically the same as the one presented in the previous paper. The difference is that at the destination there is a traffic director which, by looking at decision tables, can choose the optimal token limit and then can notify the 
traffic controller to increase (or decrease) the token limit if the buffer occupancy at the destination decreases (or increases). This information is carried by control messages

The destmation buffer is modelled as an $M / M / 1 / B$ quete The input to the network is assumed to be Poisson with rate $\lambda \in \Lambda$, where $\Lambda=\left\{\lambda_{1}, \lambda_{2}, \ldots \lambda_{h}\right\}$ is the discrete set of arrival rates.

The message lengths are assumed to be exponentally dustrbuted with mean $1 / \mu$, as are the transmission rates out of the destination buffer $\left(\gamma_{1}=\mu C_{2}\right)$

The model is formulated as a discrete time Markorian Decison Process [11(O) Furthermore, only one arrval or departure can occur at each tume slice $\delta$, that is, each message arrives at the buffer with probability $\lambda \delta$ (Bemonlli tmal) 'The avelige round trip delay is $T_{r}$ and the discrete subdivision $\hat{T}_{r}=T_{r}^{*} / \delta$

As was mentioned before, the trpffic director decules on the token limit and then notifies the traffic controller There is a random gals, however, betwern the tme the decision is made and the tune at which the new traflic aruves at the destmation 'Thes random gap makes the analysis diflocult, so th was assumed that this random gap in equal to the round trup delay $T_{r}$ Wuth this assumptuon, the decment that was marle at tume t

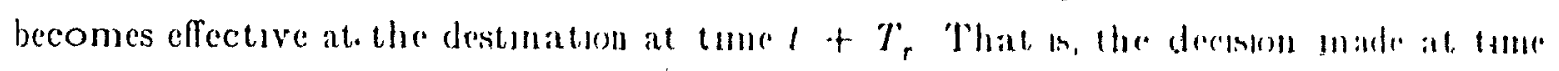

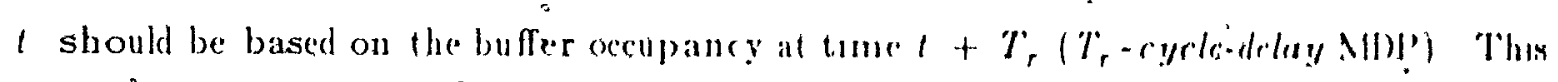
is reflected in the system state definition, which is wnll,on ats

$$
X(i)=\{n(i), \hat{\lambda}(i, 1), \hat{\lambda}(t, 2) . \quad . \lambda(t, \eta, \cdots 1)\}
$$

or in short.

$$
+\quad 1=\left\{n_{1}, \lambda_{1}{ }^{1}, \lambda_{1}^{2}, \quad, \lambda_{1}{ }^{T-1}\right\}
$$

where $6 \leq n(i) \leq B$ is the buffer occippancy at tume $t(i=t$. Al and $\lambda(i, k)$ in the mput rate to the buffer at lime $(1+k), 1 \leq k \leq T_{\text {r }}$ 
The cardinality of the set of states $S$ is given by $|S|=(B+1) K^{\left(\hat{T}_{r}-1\right)}$, where $K$ is the set of different token limits.

The set of actions of the MDP is the set of different windows $W=\left\{w_{1}, w_{2}\right.$, -.$\left\{w_{K}\right\}$. As was proven in [KLEI80], for fixed $w$ a timeout $\tau$ can be chosen to maximize the throughput of the network. The input rate set $\Lambda$ can also be considered as the set of - . actions, since each $(w, \tau)$ determines the input rate on an individual basis.

- The state transition probability for a policy $f$ from a state $i$ to a state $j$, is $p_{\imath}(f) \equiv \operatorname{Pr}[X(\hat{t}+1)=\jmath \mid X(\hat{t})=\imath$ and policy $f$ is employed $]$

A policy is a decision rule which states that given the system in state $\imath$, the $\hat{\lambda}_{k}$ is used as the arrival rate to the buffer and this rate will become effective after $\hat{T}_{r}$ time units.

The reward function $R$ is defined as $R=a \lambda-T, a \geq 0, \lambda$ and $T$ being the throughput and the delay of the system respectively,

, The immediate expected reward reflects the gain due to the throughput and the - loss due to the delay It also reflects the delay die to retransmission aftgr the message is rejected, The formula is given below:

$$
\dot{R}_{i}(f)= \begin{cases}a \hat{\lambda}_{i}^{1}-\left(n_{i}+\hat{\lambda}_{1}^{1} \hat{T}_{i}{ }^{1}\right) & n_{i}<B \\ a \hat{\lambda}_{i}^{1} \hat{\gamma}_{2}-\left(n_{2}+\hat{\lambda}_{i}^{1} \hat{\gamma}_{2} \hat{T}_{i}{ }^{1}+\hat{\lambda}_{i}{ }^{1}\left(1-\hat{\gamma}_{2}\right) \hat{\gamma}_{i}^{1}\right) & n_{i}=B\end{cases}
$$

The average reqward per unit time is given by

$$
g(f)=\lim _{n \rightarrow \infty}\left\{\frac{1}{n+1} \sum_{i=0}^{n}[P(f)]^{t} R(f)\right\}
$$

where the $[P(f)]^{t}$ is the $t$-step probability transition matrix under policy $f$. Since the action and the state space is finite and the reward function is bounded, there exists.a 
,

stationary policy under which the expected average reward per unit time is maximized. ,

This model can be solved by the policy iteration method [HOWA60]. But eveif for small values of parameters the state space grows rapidly An example given by the authors gives a very good idea of the rate of the space growth. They state that for $B=10, K=5, T_{r}=02 s$ and $\delta=4 \mathrm{~ms}$, then $\hat{T}_{r}$ becomes 50 and $|S|=11 \times 5^{18}$

- Given that at each step the method'requires the solution of $|S|$ lmear c(quations, the solution of the problem becomes computationally infeasible A heurustec solutión based on the following consideration was developed. the time for the decision to become effective can be reduced if the decision process is provided with the expected buffor occupancy at $\hat{T}_{l}$ time units later (look ahead time). With this, the new (reduced) state is defined as

$$
\text { . } X^{\prime}(\hat{\hat{t}})=\left\{\bar{n}\left(\hat{t}+\hat{T}_{l}\right), \hat{\lambda}\left(\hat{t}, \hat{T}_{l}+1\right), \hat{\lambda}\left(\hat{t}, \hat{T}_{l}+2\right), . ., \lambda\left(\hat{\imath}, T_{r}^{\prime}-1\right)\right\}
$$

An optimal policy can be found (if the new state space is not too latge) for this process. By using this policy, a suboptimal policy for the origmal policy can be found.

The decision table for the (reduced) process can be set up after the optmal policy is found. For each state of the $\left(\hat{T}_{r}-\hat{T}_{l}\right)$-cycle-delay process, the optumal foken lumit is stored. The size of this table is $N_{t b l}=(B+1) K^{-\left(T,-T_{1}-1\right)}$ By storing the current

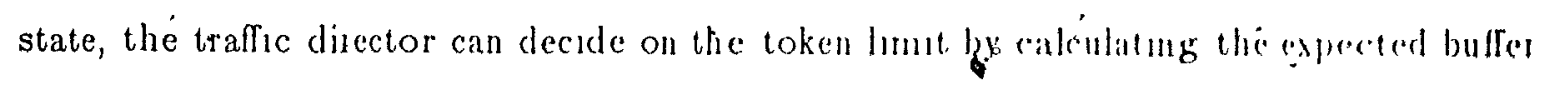
occupancy and by setting up a vector smmar to that of erpuation 43. "Tivo factors should be considered while choosing the look ahead tune, $T_{1}$. the optumality of the deci.' sions made, and the fact that the reduced deciston process should result in a rompulationally feasible solution. Large values of $T_{l}$ may result in far from optimal decisions (but computationally feasible solutions). On the other hand, small values of $T_{1}$ result in close to optimal decisions. In this case, the problem becomes mifrasible due inet rnly to 


\section{- 26 -}

the large state space but also to the multi-chain behavior of the system.

The results obtained are for values of $\hat{T}_{l}=\hat{T}_{r}-1$ (1-cycle delay) pracess. . In this case, the state of the system is obviously the buffer occupancy. These results show that the value $a$ of the reward of the system can used to control the throughput. Also the largest value of the token limit indicates the number of buffers the source node needs. The largest and the smallest token limits are controlled by the value of $a$. Furthermore, the dynamic algorithm gives slightly better results when compared to the static one.

In the third and last paper we discuss, HARB82, we find a model of the Network Control Protocol (ARPANET's former transport protocol) which assumes a reliable subnetwork. Multiple connections art portrayed (as opposed to the single ones of the previoús papers) and constant acknowledgment delays are assumed. A Markov decișion model is developed to solve the model. The presence of the curse of dimensionality is strong here too, due to the exponential growth in the number of connections of the state space. The model's structure is depicted in figure 4.2.

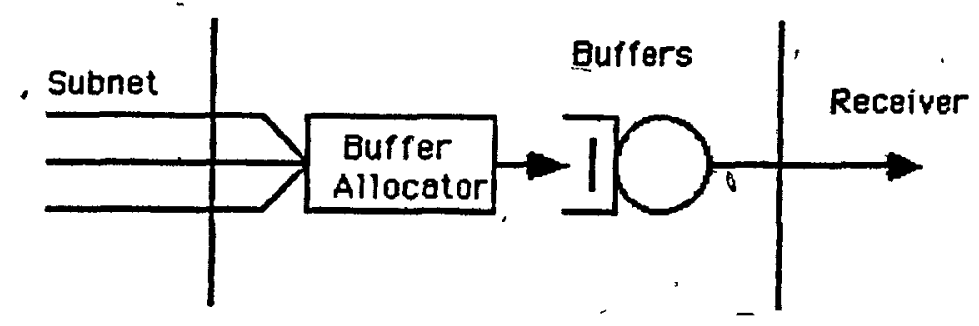

fig. 4.2

- The destination node is modelled as an $M / M / 1 / K$ queue, that is, a single server queue with finite buffer capacity, Poisson arrival rates of messages, and exponentially distributed (with mean $1 / \mu$ ) service times.

The state space of the process is defined as:

$$
S=\left\{\left\langle a_{1}^{1}, \ldots, a_{c}{ }^{1} ; m_{1}^{1}, . ., m_{c}{ }^{1} ; n^{1}\right\rangle, . .,<a_{1}^{N}, \ldots, a_{c}^{N} ; m_{1}^{N}, \ldots, m_{c}^{N} ; n^{N}>\right\}
$$


where

$a_{k}^{i} \quad$ is the allocation for sender $k$ while in state $i$

$m_{k}^{i} \quad$ is the number of messages received from $k$ while in state $i$

$n_{i} \cdot \quad$ is the number of buffers occupied in state

. c is the number of connections

$N \quad$ is the number of states

the arrival rate of messages due to sender $k$ in state $i$ is given by

$$
\quad \lambda_{k}^{3}= \begin{cases}0 & n^{2}=B \text { or } a_{k}^{2}=m_{k}^{i} \text { of } A_{k}^{2}=\dot{0} \\ \lambda_{k} & \text { otherwise }\end{cases}
$$

where $B$ is the total number of buffers at the destination host. As we can sce from this definition, the traffic from the senders is cut off when all the buffers are ocenpled. The - 'authors claim that this assumption is' justified whether the control mensigan flow on separate channels or whether they are priortized.

The cardinalıty of the state space is given by

$$
|S| \doteq\left[\left(\frac{(B+1)(B+2)}{2}\right)^{c}-B^{c}\right](B+1)
$$

- The action space $\left\langle\dot{A}_{1}^{2}, A_{c}^{i}>\right.$ represents the new allocations for marlingunection in state $i$. Whenever $A_{k}^{\prime}=a_{k}^{+i}$, then there are no control messinges issued ln the case $\boldsymbol{A}_{k}{ }^{3}>a_{k}^{\prime}$, the destination has issued an Al, message Finally, $A_{k}^{\prime} \cdot a_{k}^{\prime}$ moclels the issuance of a GVB message by the destination. The RLT mossages are not modelled.

The-immediate reward function is defined as

$$
R^{i}=-\alpha R_{d}^{i}-\beta R_{c}^{i}+\gamma \sum_{i=1} R_{i}^{i j} T_{1,}
$$

- where 
$R_{d}^{i} \quad$ is the reward per time unit due to delay in state $\cdot i$

$R_{c}^{i}$ is the reward per time unit due to control message overhead while in state $i$

$R_{t}^{i j} \quad$ is the reward per time unit due to message throughput from state $i$ to state $j$

$T_{3 j}$ 'is the transition rate from state $i$ to state $\jmath$

$\alpha, \beta, \gamma$ are normalizing constants

We can see from the above formula that a policy is penalized for the control méssage overhead and the delay, while it is rewarded for the throughput

In addition, the reward terms can be defined in the following manner:

$$
\begin{aligned}
& R_{d}=n^{i} \text { per unit time } \\
& R_{c}^{i}=r \text { If for } r \text { links } a_{k}^{i} \neq A_{k}^{i} \\
& R_{t}^{i j}= \begin{cases}1 & \text { if } n^{j}=n^{i}-1 \\
0 & \text { otherwise }\end{cases} \\
& T_{i j}= \begin{cases}\lambda_{k} & \text { if } n^{\circ j}=n^{i}+1, \quad m_{k}^{j}=m_{k}^{i}+1 \\
\mu & \text { if } n^{j}=n^{2}-1 \\
0 & \text { otherwise }\end{cases}
\end{aligned}
$$

As we mentioned before, the curse of information is present here too. An example given by the authors for 3 buffers and 2 connections, results in 364 states. Although the authors claim that the policy iteration method needs to be employed only once for each arrival rate, since in on-line applications buffer assignments are based on tables already set, the memory requirements for any reasonable set of arrival combinations will still grow quite high

The problem encountered in the previous models is not unique to these models. For any real application modelled by an MDP, the number of states is very large [HOWA78] (curse of dimensionality). Acceleration techniques [HEYM84] can be adopted in 
order to speed up the computations. Aggregation techniques [HEYM84] have also been developed in order to reduce the state space by replacing the original process with an approximate one. The problem remains, however, since for any practical application, the resulting model is very large 


\section{Chapter 5}

\section{Buffèr Allocation Algorithm,}

\subsection{Observations}

In the last chapter we presented the previous work done for the process-tó-process protocols. The models defined, although they are valuable and reasonable (despite the simplifying assumptions they rely on), are computationally and memory-wise infeasible. - This infeasibility results from both the size of the information available (combinatorial explosion) as well as the fact that special characteristics of each network are not taken into consideration

A closer look at various operational networks reveals that different kinds of services are offered for different applications (ie interactive, batch, terminal, or expedited data). Furthermore, measurements taken at different times on various networks lead to a conclusion that these categories possess some arithmetic values for the message (packet) size, connection tıme, etcetera, not considerably varying from the mean.

In the Mérit network [AUPP83] for instance, there are four different types of access (traffic) offered to the users: host-to-host interactive, direct terminal, batch, and external (execute, print) access, each having its own distinctive properties. Terminal traffic accounts for $40 \%$ of the overall measured traffic The number of by tes per packet per traffic type, the packets per connection, per traffic type, and the connection time per traffic type do not vary significantly from the mean. In addition, the average packet size for all traffic types has remained almost constant throughout the ten years of the network's operation. Similar observations can be made in other networks. 
As an example of similar observations, let us present measurements from the ARPANET.

Early measurements performed on the ARPANET [KLEIN76], show similar results for the message size (243 bit, 1.2 packets/message), and the round trip delay (90 msec). Moreover, one third of the sites generates $80 \%$ of the total traffic, while the most popular destinations receive $44 \%$ of the traffic, which is most heavy during weekday working hours.

In recent measurements of the same network, [COHN83], the performance of the NaP and the TCP is compared, at two different measuring perods, 1982 and 1983 Among other observations are: a $58 \%$ mcrease in the average messagc size, a $36 \%$ increase in the average utilization, and a $23 \%$ increase $^{-}$in the round trip delay $A$ though there are significant differences between these measurements, the ARPANET community agrees that the changes are mainly due to the conversion of the transport protocol from NGP to , the TCP, and to a lesser extent to the changes in the traffic scenario

The above observations lead us to conclude that in each network there ale-distinctive classes of traffic and traffic patterns, as well as specific characteristics such as message size and connect time for each type of traffic, that do not change drasticallyover time. Taking these factors into consideration, a forecasting (prediction) approacli to the problem seems reasonable

\subsection{The Algorithm}

The major purpose of the buffer allocation algorithm is to assign buffer space to a connection as a function of the estımated queue length for defferent calegories of traffic. The traffic categories are determined by the length of the messages sent along a 
connection. An example of such a category is interactive traffic, which would be several characters long. File transfers would be of varying lengths, depending upon the application programs run at the hasts Furthermore, buffer space assigned to a category is accessible to any category of smaller message length (that is to say, smaller meśsages have higher priority). It is assumed that statistics on the frequency of different length messages are collected by the network. It is also assumed that the transport level protocol in each host (gateway) keeps statistics on the queye length for each category of network traffic.

In the context of OSI-style networks, each connection can then identify itself as belonging to a particular category, èg., interactive or file transfer For a datagram style gateway, it would be necessary for the gateway to determine the packet length of each packet from information contained in its header, or by directly determining its length.

The algorithm may be divided into two piarts.

- Prediction (via exponential smoothing)

- Allocation (and deadlock avoidance)

In the prediction portion of the algorithm, the expected queue length for the different categories of traffic is forecasted.

- During a measurement period $(t-1)$ of fixed length, the queue lengths $z(t-1), i$ on the part of each category $i$ of traffic are accu'mulated. Af the end of the measurement period, the numbers accumulated for each of thecategories are inserted into the exponential weighted moving average formula (EWMA) which we employ to predict the future requests for each category. The EMWA formula is given by

$$
\hat{Z}_{l, i}=\alpha^{*} z(t-1), i+(1-\alpha) * \hat{Z}_{(t-1), i}
$$

where 
$\hat{Z}_{(t-1), i} \quad$ is the previous estimate of the queue length for category $i$ up to period $(t-1)$,

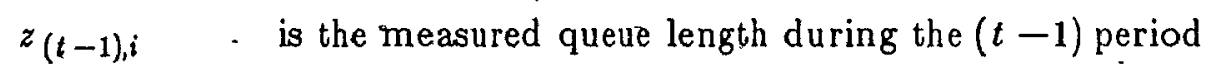

$Z_{t ; i}$

is the predicted queue length for period $t$

$\alpha$

is a parameter chosen between 0 and 1 . Chosing $\alpha$ closer to 0 emphasizes the past, while chosing $\alpha$ closer to 1 emphasizes more recently made measurements. The value of the $\alpha$ can be estimated experimentally, that is, by comparing forecasted and observed values.

- A brief discussion. on tıme series analysıs is given in chapter 3 . The interested reader, however, will find an extensive and, comprehensive discussion of the topic in BOX76.

In the allocation portion of the algorithm, the maximum buffer space allocated to each category $b_{t, i}$ is determined. The-allocation for each category $i$ is then the floor of the predicted queue length for each category. That is, .'

$$
b_{t, i}=\left\lfloor\hat{Z}_{t, i}\right\rfloor \text { 。 }
$$

In the case that there is buffer space left over, this space is then made accessible to -all connections on a FCFS basis.

Should there be a tıme period during which certain traffic is sporadic while other traffic is very heavy, the queues for the sporadically arriving traffic will not build up, while those for the heavy traffic will monopolize the allocatable bulfer space In order to prevent starvation of the sporadic traffic, a minimum allocation is granted to each category. This minimum allocation can be netwórk and/or implementation dependent. A minimum allocation equal to the sporadic traffic's average message length gives best results. A more detailed discussion of this policy can be found in chapter 7 , results.

In the event that the receiving transport station buffers data, a deadlock avoidance algorithm is combined with the allocation portion of the algofithm so that the very real 
possibility of reassembly deadlock is avoided The destination host buffers the incoming segments of data until the last segment arrives and then hands off the entire message to the receiving process Since buffer space may be allocated to partially received messages (depending upon therr length), it is possible that the entrre complement of space allocated by the host to the transport protocol is occupied by partially received inessages

- Since none of these messages may be delivered to the receiving processes, we have the classic reassembly deadlock 1

- To'avord this deadlock, we employ the following algorithm.

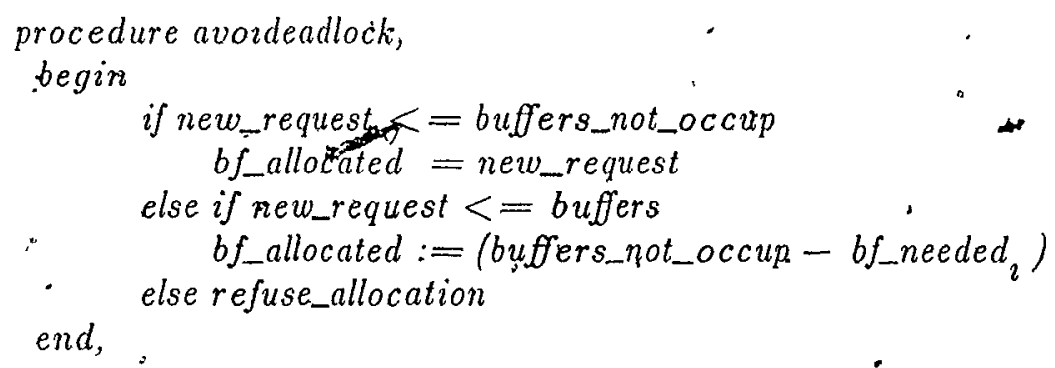

where

néw_request

buffers_not_occup

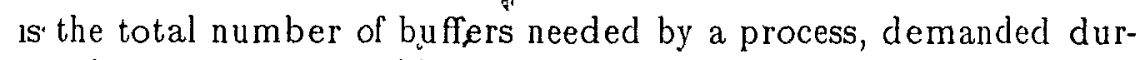
, ing the connection establishment

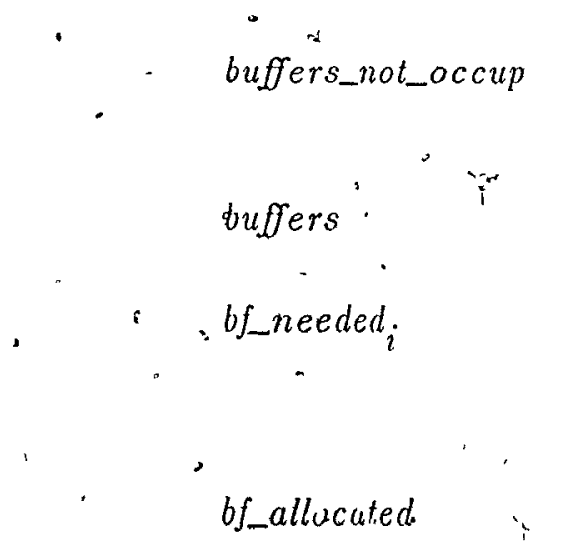

Is the number of buffers which are not occupled at the time the new request is made

is the total buffer space at the recelver

Is the number of buffers needed, in order to be completed, by the " 2 -th requ hach was partially satisfied (requests are belled in increasing order)

bf_allucated.

is the current number of buffers allocated to a new request

We will prove the following proposition:

A request, once accepted, has an assured departure.

\section{Proof}

For an incoming request $\imath+1$, two situations may occur. 
either $b f_{-}$needed $\dot{i+1}_{+1}=9$ in which case the request is satisfied by the first condition of the algorithm and eventually will depart, or the new_request $>$ buffers_not_orcup. In this case we claim that $b f_{-}$needed ${ }_{i+1}>b f_{-}$needed ${ }_{2}$ The proof for this is

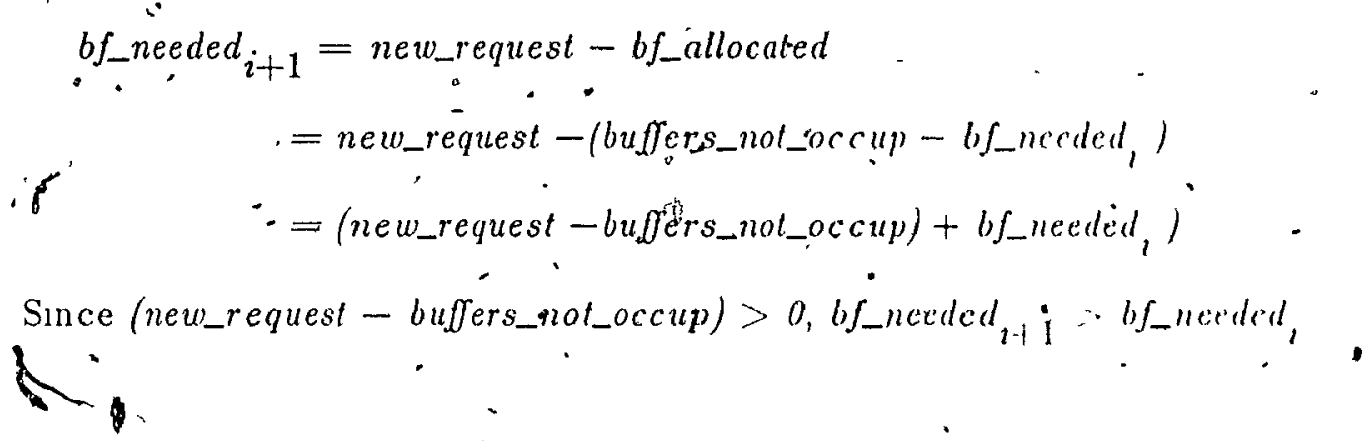

At the arrual of the $(1+r)$-th (partially satisfied) jol), the algot thm guaranteres that

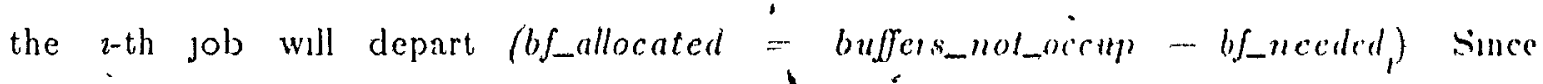

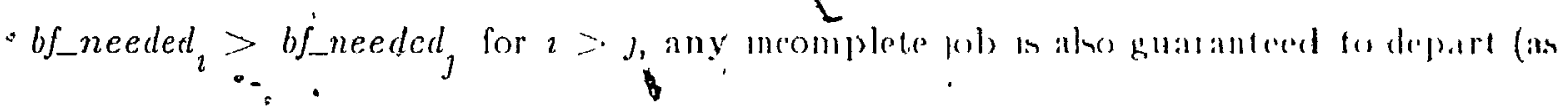

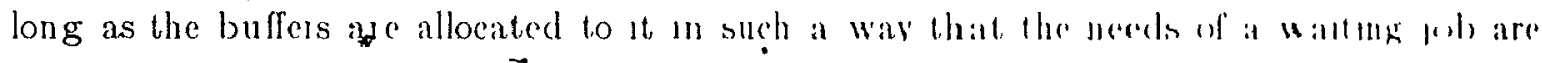
completely satisfied before the allociation prgeceds to the new job)

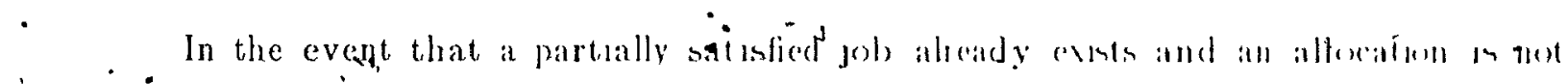

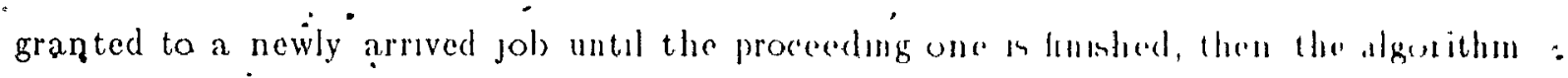
- operateg on a fcls basks."

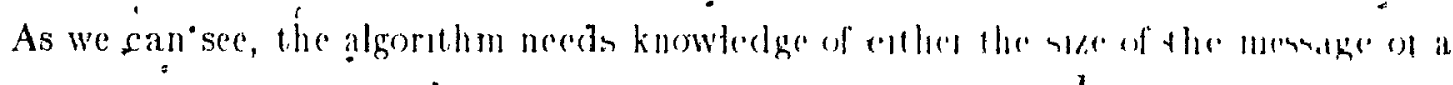

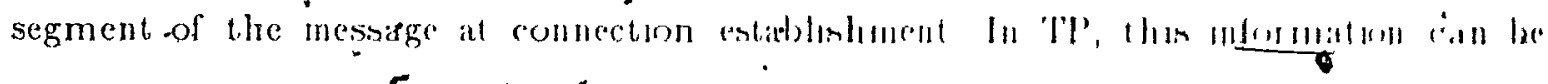

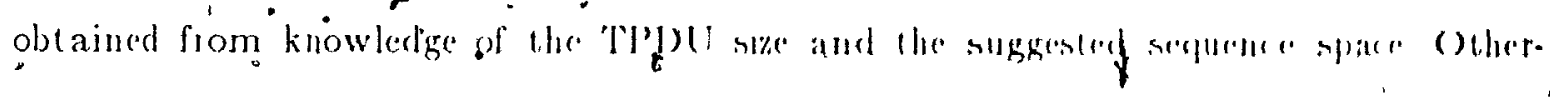

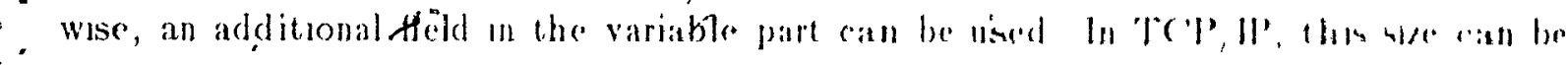
obtaned directly fróm the II' header 


\section{Chapter 6 .'.}

Simulations

\subsection{Network Mọdel}

Figure 6.1 below portrays the network structure of the model employed to evaluate the allocation algorithm.
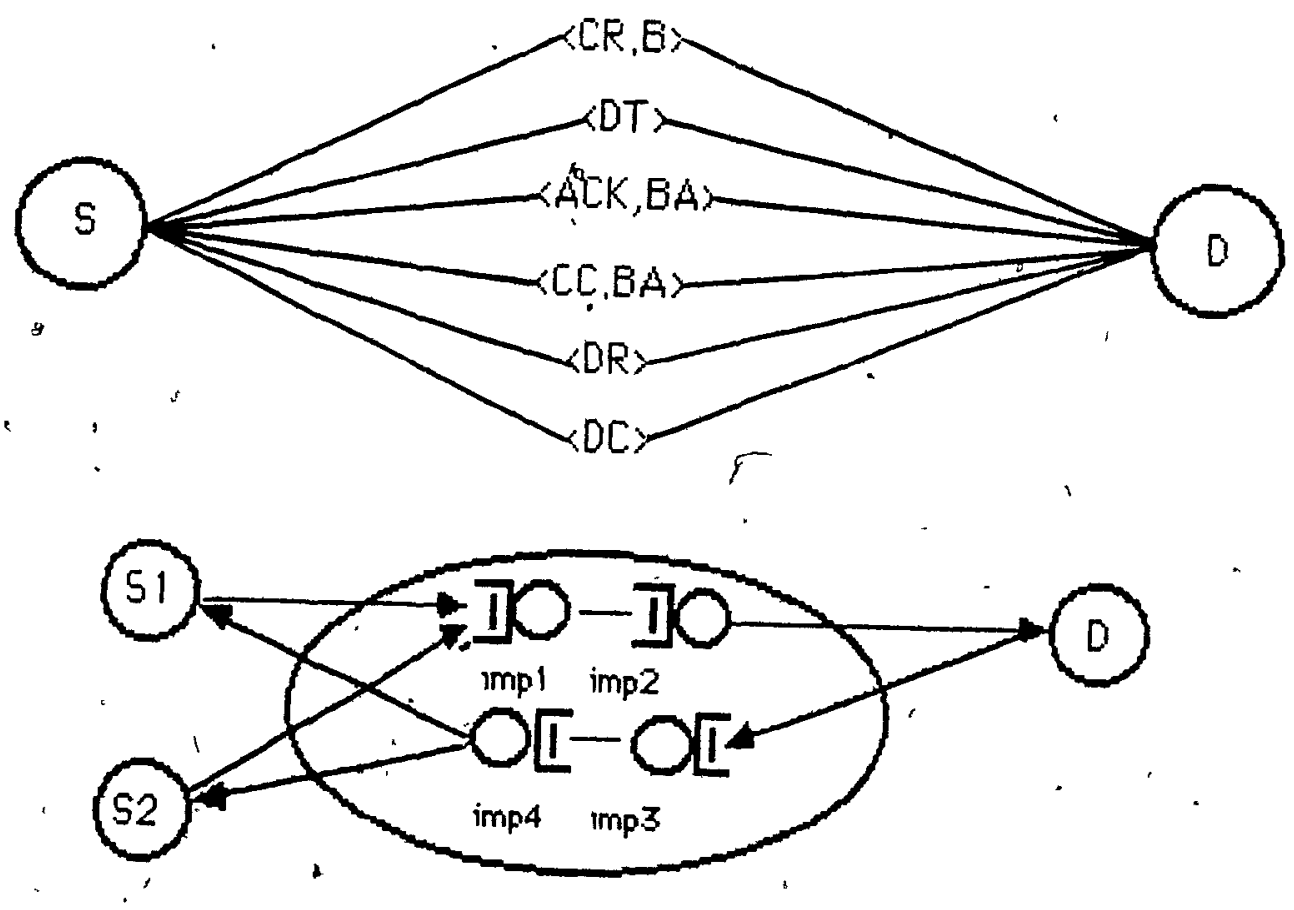

fig 6.1 TP Model

As can be seen from the figure, we represent two transport, level connections $\left(C_{1}\right.$, $C_{2}$ ) originating at sending stations $S_{1}$ and $S_{2}$ and terminating at receiving station $D$. The messages on both connections travel through the network along the top route - through switches (imps) 1 and 2 where they are passed to the receiving transport station. Acknowledgements return along the bottom path via switches 9 and 4 . 
Message lengths on both connections are chosen from exponential distributions. The mean message length on $C_{1}$ is 512 bits, while on $C_{2} 60 \%$ of the messages are 1024 bits, $20 \%$ are 2048 bits and $20 \%$ are 4096 bits. Acknowledgements are 80 bits long. The buffer size at the receiving station is 512 bits, while there is a total of 16 buffers.

We assume that the link data rates are $96 \mathrm{Kbps}$ and that the lines are free of errors The data pates from the destination host to the end user are assumed to be 2.4 Kbps

Access to the network is controlled vala a fixed $(=8)$ wudow mechamm Whithin the subnet we employ, a fixed timeout retransmission protocol between switches in the event of buffer overflow at the destination switch.

Figure $\dot{6} 1$ also depicts the part of OSI ans 4 transport protocol ('Tl't) whichs we simulate As can be seen, connection establishnent, is acromplished by crelange of a

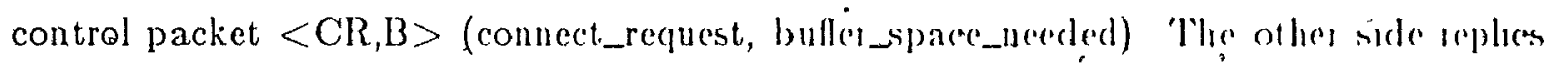
with a $<\mathrm{CC}, \mathrm{BA}>$ (connect_confirm, buffer_space_allocated) m the event that 11 agrees to open the connection, or with $<D R>$ (disconnect_request) if it doesn't In the latter case, the sender tries to open the connection later by employmg the same secpuciner of control packets

Once the connection is established, the sender sends the datid pachets - Dot . The recelving station then replies with $<A C K, B B A$. (acknowledgement, biffreallocited).

In order to termmate the connection, the sender employs a . DR. - and the recerver replies with $\angle \mathrm{DC}>$ (disconnect_confirm)

In order to evaluate the algorithor, we compare its performance to fCPS under varying traftic loads and for different values of a The farameter' a controls the emphasis on the most reent measurement period - as a, mereasen, the sugnifienuse of the most recent period increasen 


\subsection{Simulation Model}

As indicated in the introductory chapter, we employed the Performance'Analyst's Workbench System (PAWS) as our simulating tool in evaluating different buffer allocatión algorithms [PAWS].

PAWS is a simulation language which supports high level primitives such as polling or last come first served queueing policies, best or first fit memory management disciplines, or hyperexponentral, erlangian distributions, thus releasing the user from the detalls of coding, and permitting concentration on the model itself The language also encourages and supports the pictorial representation of the model, or Information Pro.cessing Graphs (IPGs) as they are called in PAWS, as PAWS translates and evaluates these-IPGs directly The IPGs consist of nodes and edges. Nodes represent the place in which information is processed, while edges signify the information flow from one node to the other. The basic unit of information is the transaction. A transaction is data that can be created, processed, moved from one node to another, or destroyed.

Figure 62 portrays the IPG of our model, and consists of three parts:
a) the source host
b) the communication subnet
c) the destination host

Our description is divided accordingly.

\section{a) The Source Host}

Transactions representing messages are created at node GEN according to a Poisson arrival Process Node CTLO serves the purpose of controlling the input, in order to avoid saturation of the system. At node MSGPARM, a message length is assigned to 
each transaction. After this node, a connection establishment with the destination node is attempted by creating a control packet at node MSGSPLIT. At node PPARM each packet is assigned its own parameters. The original transaction waits at node MSGTO until the reply of the other side returns. The nature of the reply causes the origmal message to be split into more packets (nodes PFORMAT and MSGSPLIT) or to wat for buffer space allocation At node MORETPDU, the message is split into smaller units, which are transmitted separately, in case the destination can not store (reassemible) the complete message

Each packet leaving the MSGSPLIT nod"e goes through the node ALLW1 or ALLW2 These nodes are used to simulate a fixed window flow control policy at the network level. Each packet should grasp a credit, before its admittance tó the network, which is returned back through node RELW upon the recelpt of the acknowledgment." Next in the row is the node BACKUP There, a copy of each packet is kept for retransmission in case the acknowledgment doesn't arrive prior to the expuration of the timer The node TIMER is used for this purpose.

Finally, the SENDER nodes represent the communication links from the sender processes to the network

In addition, throughout the diagram the COLLECT nodes, in conjunction with their corresponding $\mathrm{BH}$ nodes, represent the exit of a transaction from the system.

\section{b) The Communication Sủbnet}

The IMP nodes represent the links from one switch to another in the network $A$ finite buffer space is associated with each link, hence packets are retransmitted in the event that no acknowledgment is received after a time-out period. This is handled by the 


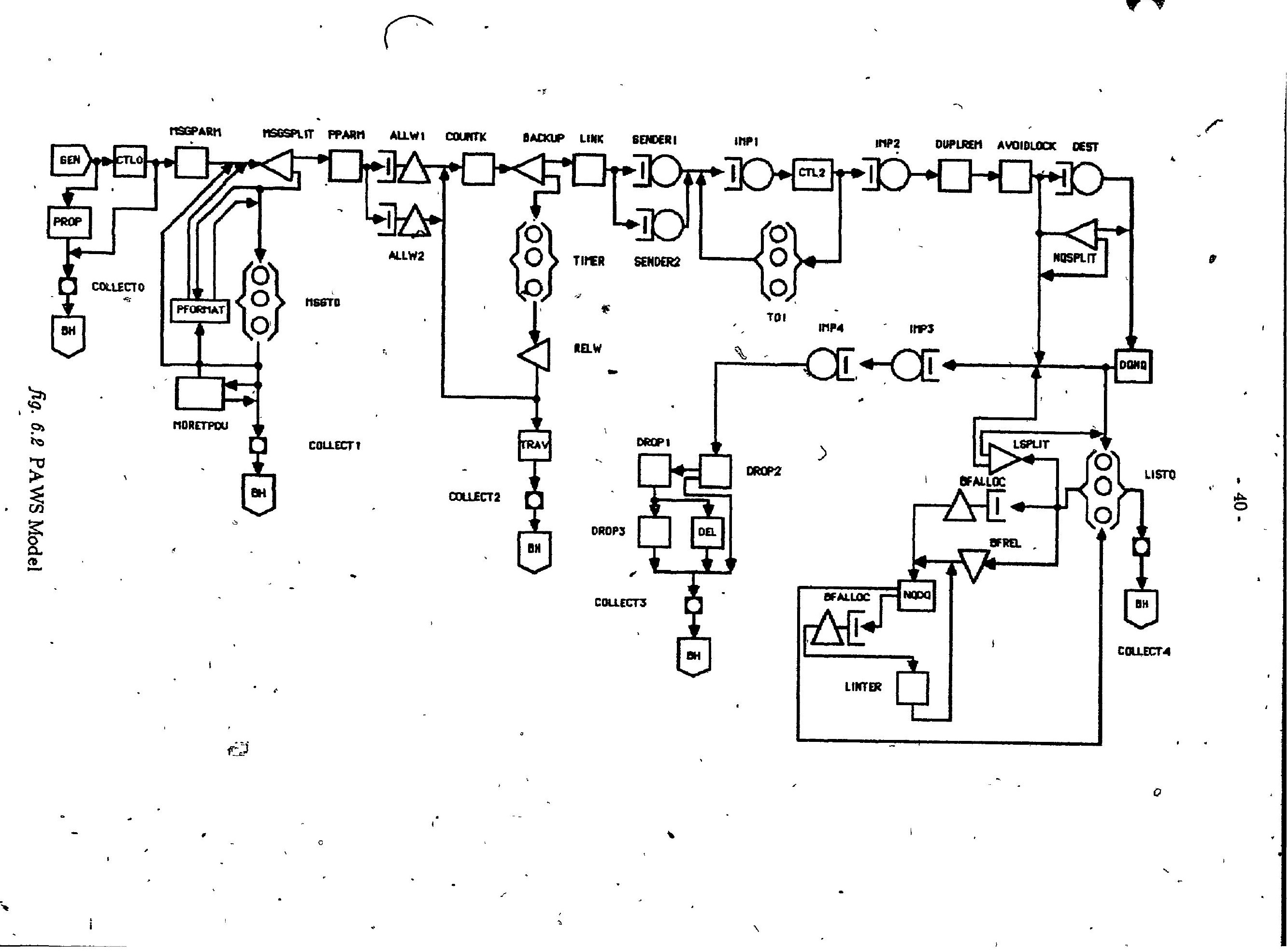


CTL2 and TO1 nodes IMP nodes 1 and 2 represent one direction of data flow, while IMP's 3 and 4 represent the reverse direction of flow. The DROP and DEL nodes serve the purpose of notifying the sender of the successful delivery of a packet or a message. They accomplish this by delivering (to the sender) the control packets.

\section{c) The Destination Host}

This is where the buffer allocation algorithm resides. The DÜPLREM node serves the purpose of removing the duplicate packets, whlle node $A_{0}$ VOIDLOCK keeps track of the incoming requests for each message category We simulate two calugorles of messages, long and short, single TPDU's and multiple 'TPDU's The exponential smoothng and split of the buffer space is dong at node PROP The dendlock nvoidance algonthm resides at node AVOIDLOCK, while blffers are allocated and released through nodes. BFALOC and BFREL respęctıvely. Nodes LISTO, DQNQ, and NQDQ kecp track of the current buffer space allocated and the relcvant detals for cach packet/messingo Acknowledgment, contıol and allocation packets are returned by the NQSPLIT, ISLLIT, and LINTER nodes Finally, completely received messages are dolivered to the. end user through the node DEST. 


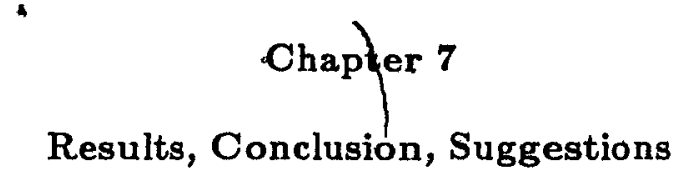

\subsection{Results}

As previously indicated, the buffer allocation algorithm was compared (when appropriate) to the one adopted by TCP and TP (which is called here fcfs) The simulation package employed in order to carry out the simulation wgs PAWS, and was run on a VAX 11/780 The average simulation length for each run was three CPU hours. The results presented constitute a small but representative subset of the runs. Averaged batch values are presented

Our results, depicted in the graphs at the end of the chapter, are for

a) different interarrival rates (graphs $7.1 \mathrm{a}$ to graphs $7.1 \mathrm{j}$ ),

b) varied minımum allocations for the buffer space, a factor which becomes relevant under extreme conditions, (graphs 7.2a to $7.2 \mathrm{~b}$ )

c) different values of $\alpha$ (graphs 7.3a to graphs 7.3h).

The performance values of interest are the end-to-end delay and throughput. The graphs 71 and 7.2 depict four curves, one for each category of traffic under each algorithm, while the graphs 7.3 depict two curves, one for each category. The letter $E$ in the graphs stands for exponential, the $\mathrm{F}$ for fcfs, the $\mathrm{S}$ for short, and the $\mathrm{L}$ for long.

\section{a) different interarrival rates}

- For graphs 7.1 the average message length for long messages is 4.6 packets/message 
while the short messages have 1 packet/message. Each packet is 512 bits. The exponential smoothing paraméter $\alpha$ has the value of 0.6 , while the measurement period during which the statistics are accumulated is 40 seconds. The interarival rates for short messages $\left(\lambda_{1}\right.$ seconds) and for long messages $\left(\lambda_{2}\right.$ seconds) take values from the set $\dot{\Lambda}=\{0.1,0.5,10,15,2.0\}$ This set was chosen in order that all possible input rates, -from high utilization $(\sim 90)$ to low utilization $(\sim 30)$ may be covered The recciving station. has 16 buffers (512 bits each).

1) End-to-End Delay (graphs 7.1 a to graphs 7 1e)

Starting from graph $7.1 \mathrm{a}$ where $\lambda_{1}=01$, we notice that when the utilization is heavy $(\sim 96 \%)$ there is a small gain $(\sim 8 \%)$ for shot t messages which dimmishes to fcfs at low $(\sim 62 \%)$ utilization On the other hand, long messages pay the corresponding price up to $\sim 15 \%$

In the next graph $(71 \mathrm{~b})$ where $\lambda_{1}=05$, we observe that for $\lambda_{2}=01$ and $\lambda_{2}=0.5$, there is a small gamn for short messages whlle longer messages again register a corresponding loss. At the point where $\lambda_{2}=1.0$, the gain is becommg noticeable ( $\sim$ $21 \%)$ and the there is still a gain, however, since the values are small and the variances are large this difference is not significant.

In the graphs $(7.1 \mathrm{c}, 7.1 \mathrm{~d}, 7.1 \mathrm{e})$ where the short messages arrive at a lower rate $\left(\lambda_{1}\right.$ is $1.0,1.5$, and 2.0 respectively), we notıce that there are points where the gaili becomes much more visible while long messages seem to gan as well

The phenomena are explained thus:

When both short and long messages arrive at a very high rate, there is an opportunity for short messages to ayal! themselves of their required buffer space even under 
fcls. This is why the gain under the algorithm is hot impressive.

There are certain combinatıons of interarrival rates (especially when short messages arrive at a relatively lower rate as compared with longer messages) where under the fcfs policy there is a distinct possibility for shorter messages not to recelve buffer space due to their being overwhelmed by the longer messages' demands On such occasions the algorithm shows its value, in that at all times it guarantees buffer allocation to the shorter messages (in varying amounts according to the predicted buffer occupancy) while . at the same time, not preventing them from occupying empty buffer space in the section set aside for longer messages.

The gain noticed for the longer messages results because better buffer management is accomplished by the reservation algorithm.

2) Throughput (graphs $7.1 \mathrm{f}$ to graphs $7.1 \mathrm{j}$ )

We notice an insignificant difference between the reservation algorithm and the $\mathrm{fcfs}$ (a very small gain for short messages accompanied by a very small loss for longer messages). This is indicative of the algorithm's farness. The small loss for the longer messages is the result of the gain, in both the delay and throughput, for the short messages.

b) varied minimum allocations

Graphs $7.2 \mathrm{a}$ to $7.2 \mathrm{~b}$ deal with the (extreme) case where one traffic stream is sporadic and the other is frequent. In this case, the prediction indicates that all buffer space is taken by the heavier traffic stream, while the other stream experiences starvation. The algorithm was tested for different values of minimum allocation; one buffer, two, and three (shown in the graph labelled mintpdu) We assume that $\lambda_{1}{ }^{\prime}=0.005$, $\lambda_{2}=5.0, \alpha=0.6$, and the total number of buffers is equal to 4 . Finally, the average 
message length for sporadic traffic is equal to 3 packets/message, while short messages have $1 \mathrm{packet} / \mathrm{message.}$

We observe in the graphs that with the value of minimum allocation being equal to one, the sporadic messages suffer a big delay and a corresponding degradation in throughput, since the small allocation necessitates their being split in to muluple onepacket mesșages.

When the minimum allocation is equal to two, the loss for sporadic messages in delay. and throughput is almost halved Contrary to what one might in short mêssäges exhıbit a general, albe1t small, improvement under this type of allocation. This becomes obvious when one realizes that the longer messages are now split into fewer packets, which translates into fewer control packets and their spending less time occupying the avarlable buffers.

With the minimum allocation set at three, the results show neither signficant improvement nor deterioration over the fcfs. Ih general, large average message lengths for sporadic traffic and small values for minimum allocation create problems all around. A value of minimum allocation close to average message "length seems to present the most de'sirable situation.

\section{c) different values of $\alpha$}

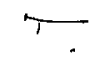

This section focuses on the effect upon the results when different values of the exponential smoothing factor $\alpha$ are introduced. Specifically, the values used are: $0.1,0.3$, $0.6 ; 0.9$. The average message length for each category, as well as the number of buffersat the receiving station; is the same as in graphs 71 .

For graph, $73 \mathrm{a}$, where $\lambda_{1}=0.5$ and $\lambda_{2}=0.1$, we notice that under high utilization 
( $\sim 95 \%$ ), the higher values of $\alpha$ give better results for delay. The same holds for the throughputs portrayed in graph 7.3e. The results for both are explained by the fact that under higher values of $\alpha$, the algorithm reacts faster and naturally gives-better predictrons. In graphs $7.3 \mathrm{~b}$ and $7.3 \mathrm{f}$ (delay and throughput respectively), whare $\lambda_{1}=0.5$ and $\lambda_{2}=0.5$, the pattern mentioned above is repeated.

Turning our attention to graph 7.3c, where $\lambda_{1}=0.5$ and $\lambda_{2}=15$, we see that under lower utilizations $(\sim 75 \%)$, smaller values of $\alpha$, ie. $\alpha=03$, give better results for delay: The same is true for the throughput of the long messages (graph $73 \mathrm{~g}$ ), while the results for throughput of short messages-do not differ significantly Furthermore, simulation runs attempting different values of $\alpha$ for each category of messages (not shown in the graphs) showed that, thalue of $\alpha$ for short messages is important while the corresponding $\alpha$ value for the long messages is irrelevant. This is explained if we note that 1 ) the higher the value of $\alpha$ for short (frequently arriving) messages results in a larger allocation, and that 2) short messages can acquire and use th tially allocated for the large messages, but not vice versa. Hence, a high value for $\alpha$ predicts a larger allocation which is taken from the longer messages' space. Consequently, small välues of $\alpha$ give better results. This points out the importance of selecting the values of $\alpha$ based upon arrival rates and buffer utilization.

Finally, from graphs $73 \mathrm{~d}$ and $7.3 \mathrm{~h}$, where $\lambda_{1}=1.0$ and $\lambda_{2}=2.0$, we notice that for low utilization $(\sim 38 \%)$, 1 does not appear necessary to optimize the value of $\alpha$ since the variances in all cases are quite large and the delays.are small.

We conclude from these graphs that:

- at low to moderate utilizations the choice of $\alpha_{1}$ does not seem to significantly affect the results

- at high utilizations higher values of $\alpha$ seem to give better performance but different values of $\alpha$ should be assigned to the categories depending upon their arrival rates. 


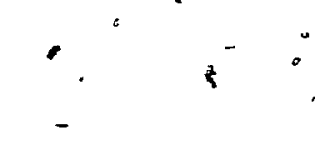

Simulation runs for connectionless transmission of short messages showed no sighificant difference over the connection-öriented transmission. This is explained if we take into consideration that the gain resulting from the elimination of the connection establishment phase can.be timinished due to the extra qqueuing delay of the complete' messages.

\subsection{Summary and Conclusion}

1

We have presented in "this thesis a buffer allocation algorithm for use in a transport protocol such as ISO's TP class 2,4 or TCP. The objectıve for the algorithm was that of obtaining a fast response to short messages while mantainng an acceptable throughput for longer messagés.

The algorithm allocates buffer space as a function of the estimated queue length for each category of traffic (categories are user defined) thereby ensuring fairness on its part. Furthermore, categories of smaller message length can acquire and use buffer space, if available, initially allocated to categories of longer message length, but not the other way -

around. This way, it is guaranteed that for short messages the algorithm operates at least as well as the one using fcfs discıpline ${ }^{\circ}$ The prediction was done via exponential smoothing $\dot{A}$ deadlock avordance algorithm was also employed to avoid tlfe possibility of reassembly deadlock.

The algorithm was compared by simulation to a fcfs discipline. Different traffic scenarios, exponential smoothing factors $(\alpha)$, and buffer spaces were examined in the course of the simulations

- Under heavy traffic conditions, and'especially when short messages arrive at a relá. tively lower rate as compared with longer messages, the algorithm resulted in a gain in 
the end-to-end delay, and throughput for shorter messages as compared to fcfs. This was the result of reservations being matde for the shorter messages, thus avoiding dominance of the buffer space by longer messages $\Theta \dot{n}$ the other hand, there is a degradation in the performance for the longer messages which pay the price for the gain of the shorter messages. Under low utllization the algorithm fluctuates around the levels achieved by the fcfs strategy:

- In the case of sporadic (unpredictable) traffic, the algorithm degenerates to fcfs when the values of minımum allócation are close to average message length for this traffic.

- The choice of $\alpha$ should be made according to utilization For low utilization, the choice of $\alpha^{*}$ is largely irrelevant. At high utilizations, larger values of $\alpha$ seem to give - better results Careful consideration should therefore be given to chosing $\alpha$ for each category as a function of the arrival rate of messages in each category as well category as a function of the arrival rate of messages in each category as well

\subsection{Suggestions for Future.Work}

The EWMA formula, although it seems a reasonable approximation, is by no means the optimal one for all possible traffic scenarios It is up to the network monitor to collect the data and find the appropriate ARIMA model (or mòdels) and its associated parameters $($ le $\alpha)$ that best fits the data

An extension of the algorithm can be used in a gateway between different networks In thís" case, the algaithm should account for different transmission media as well as for different categories of networks 


\section{3}

$-8$
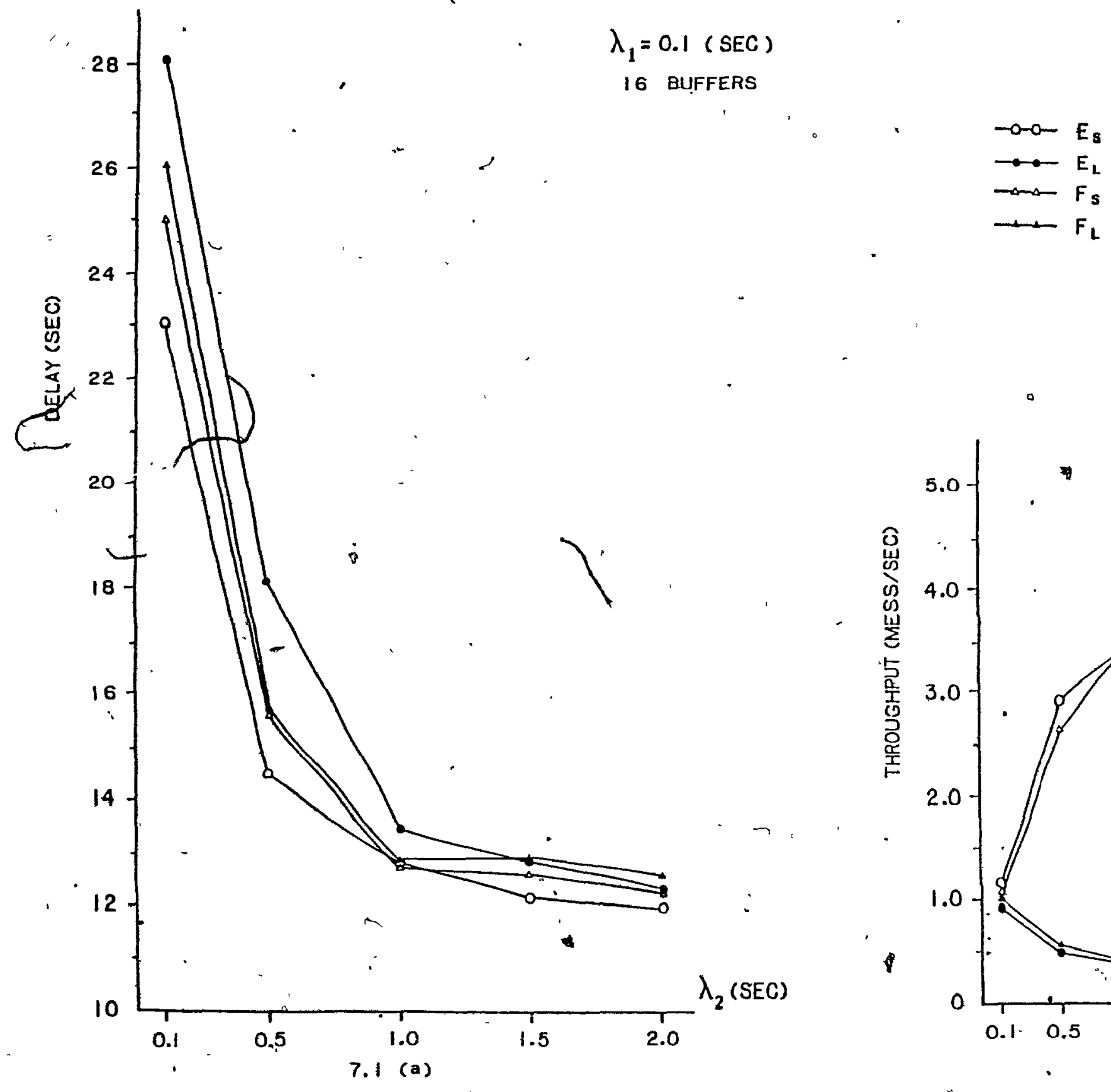

:

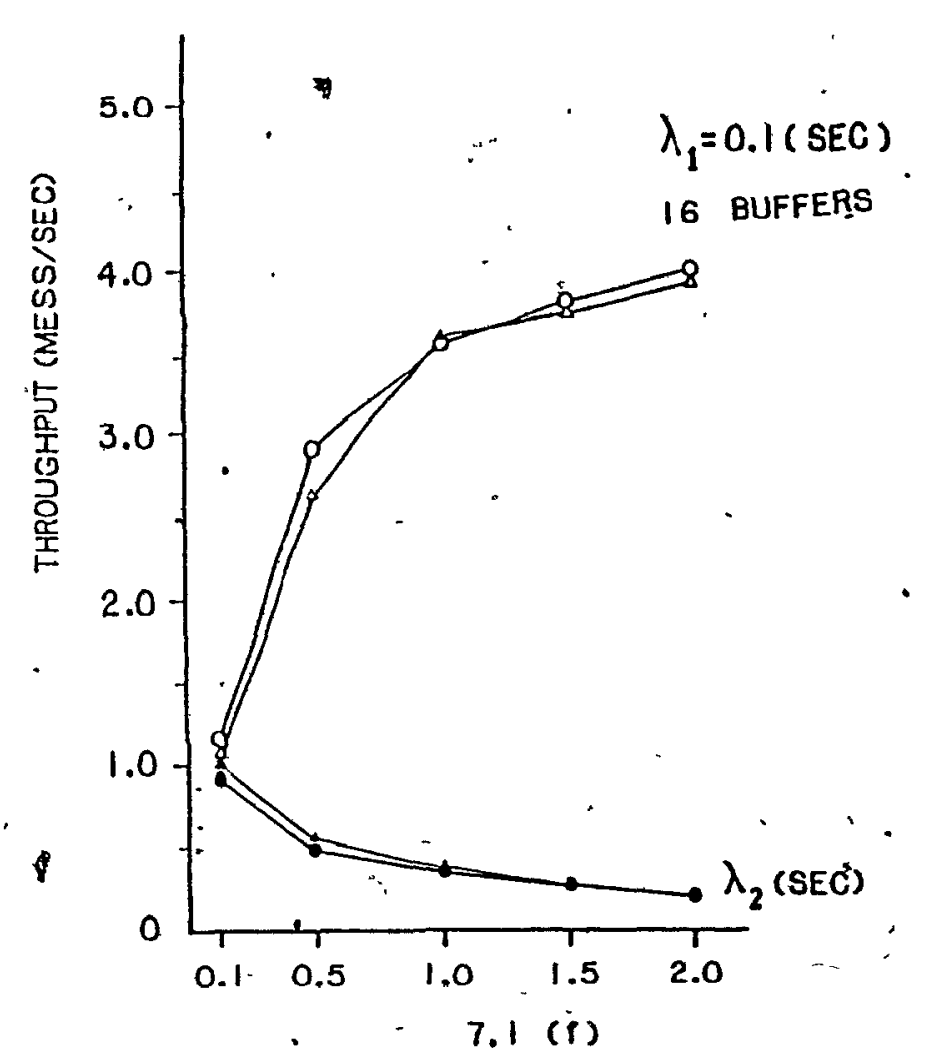




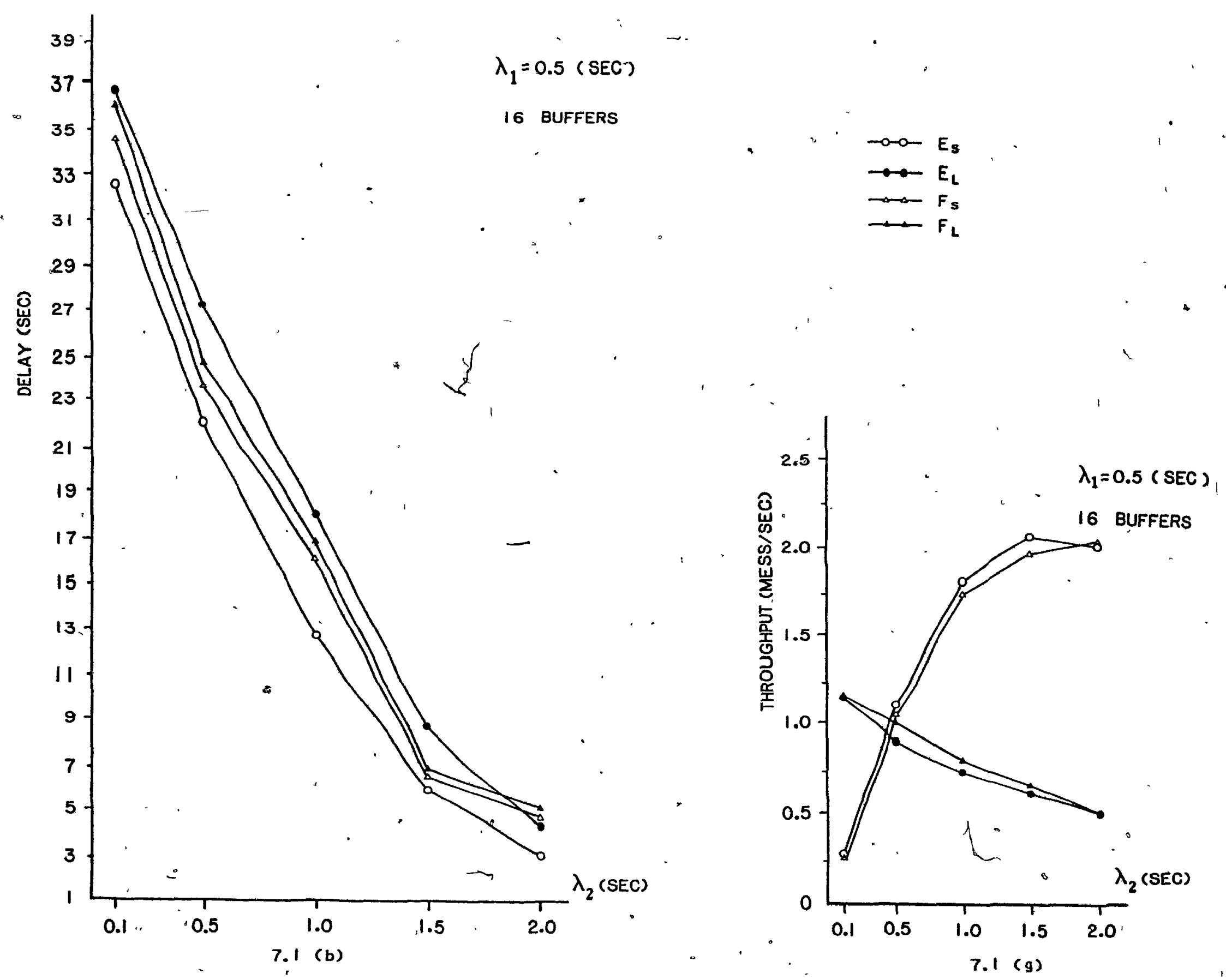



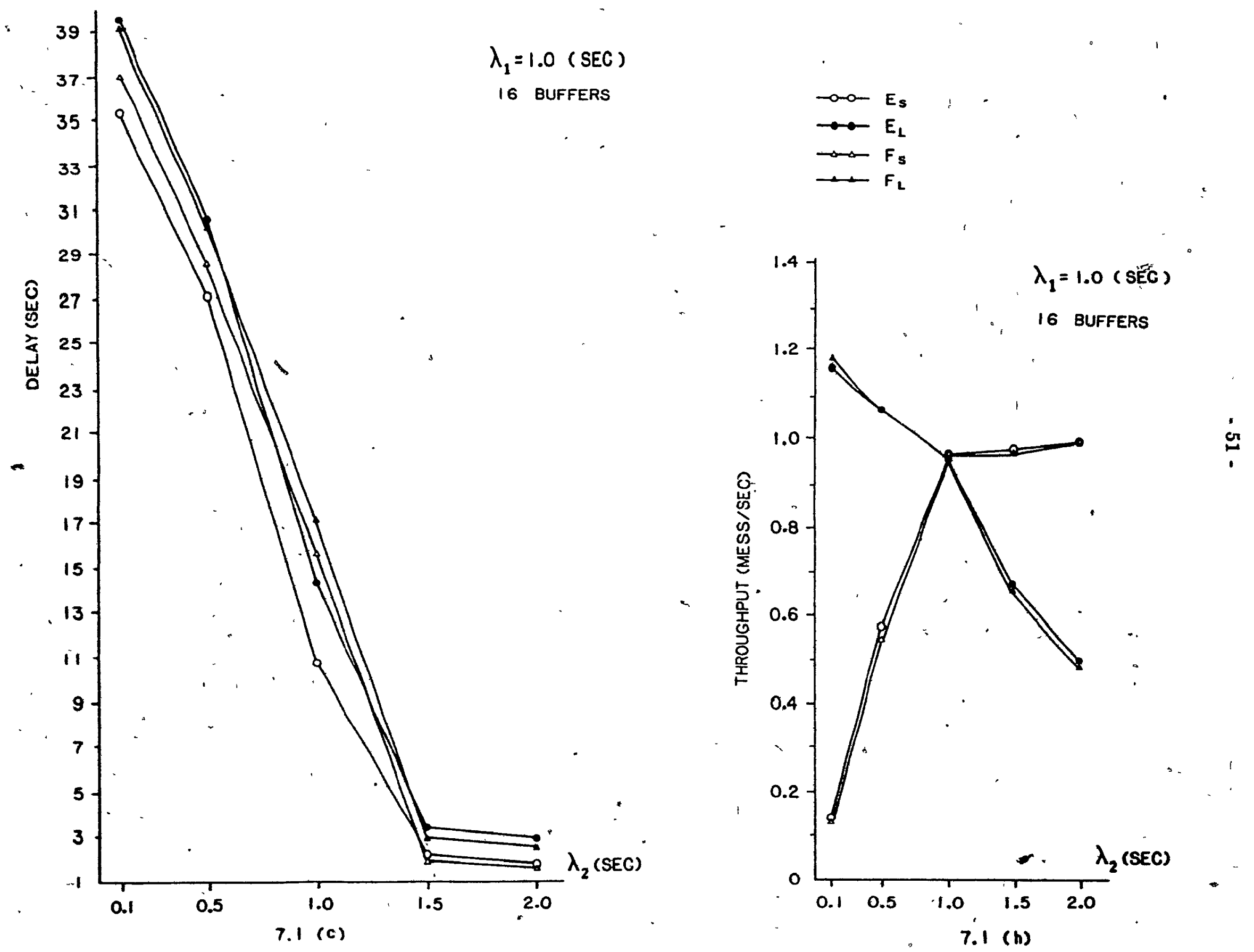

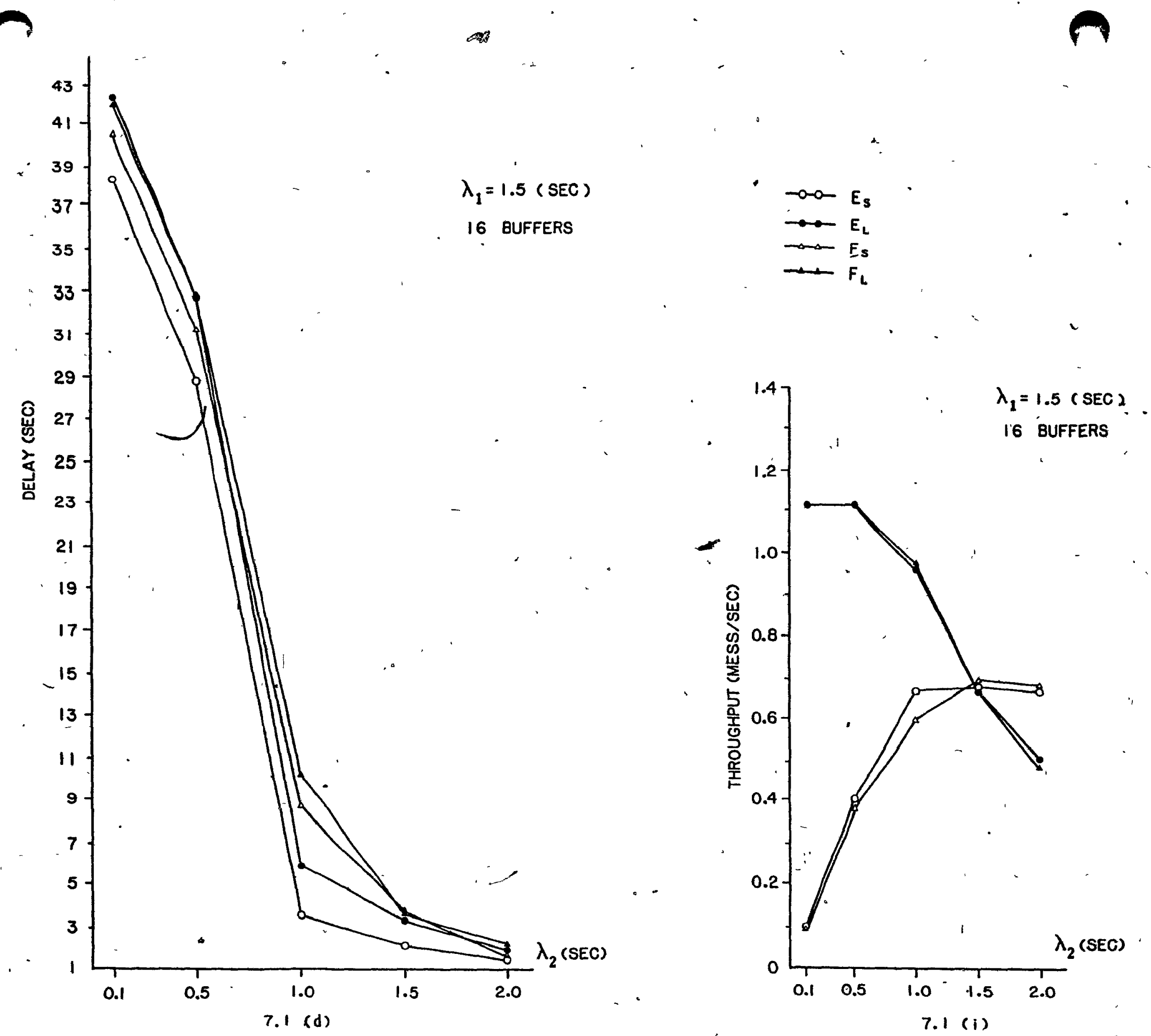


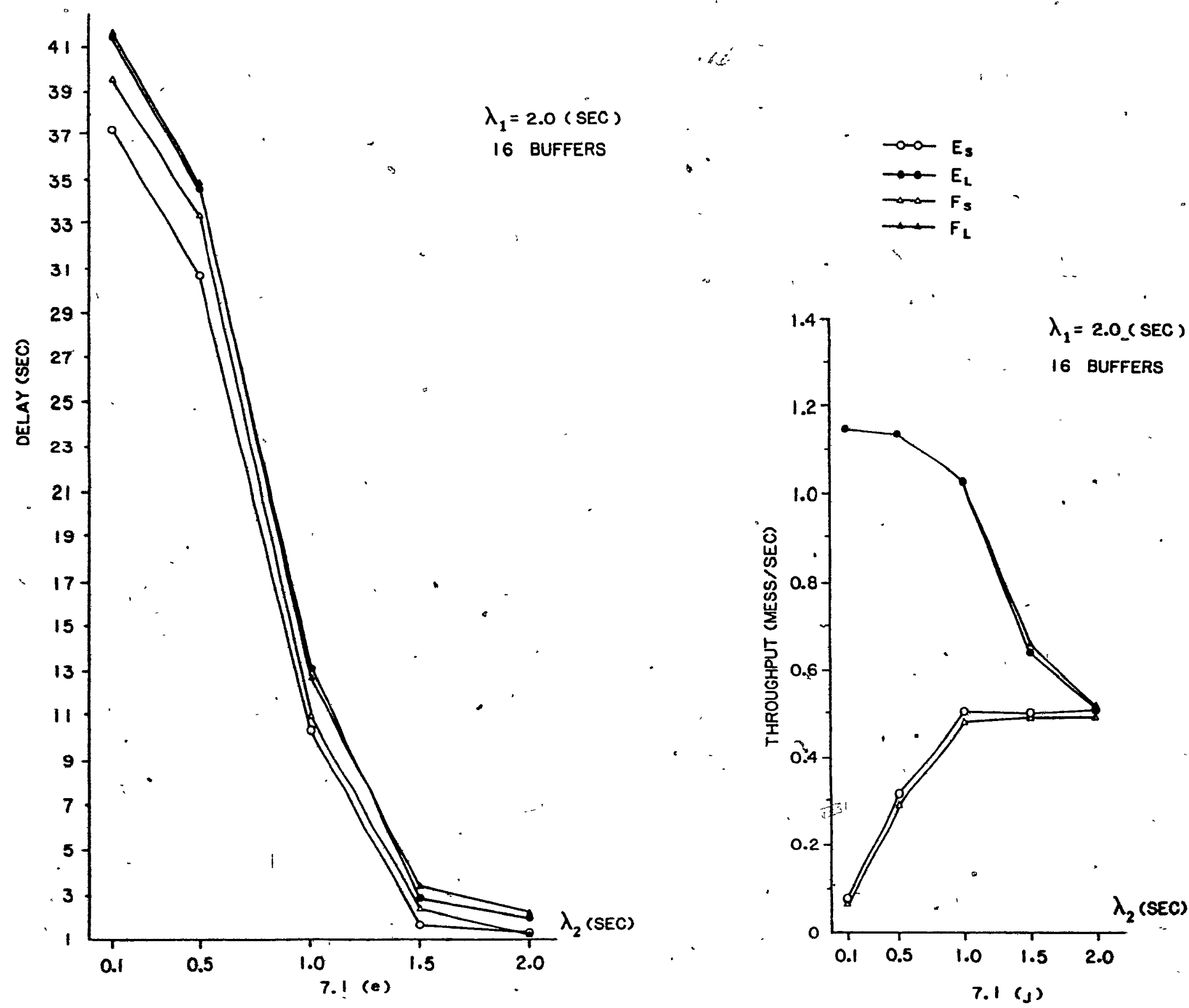



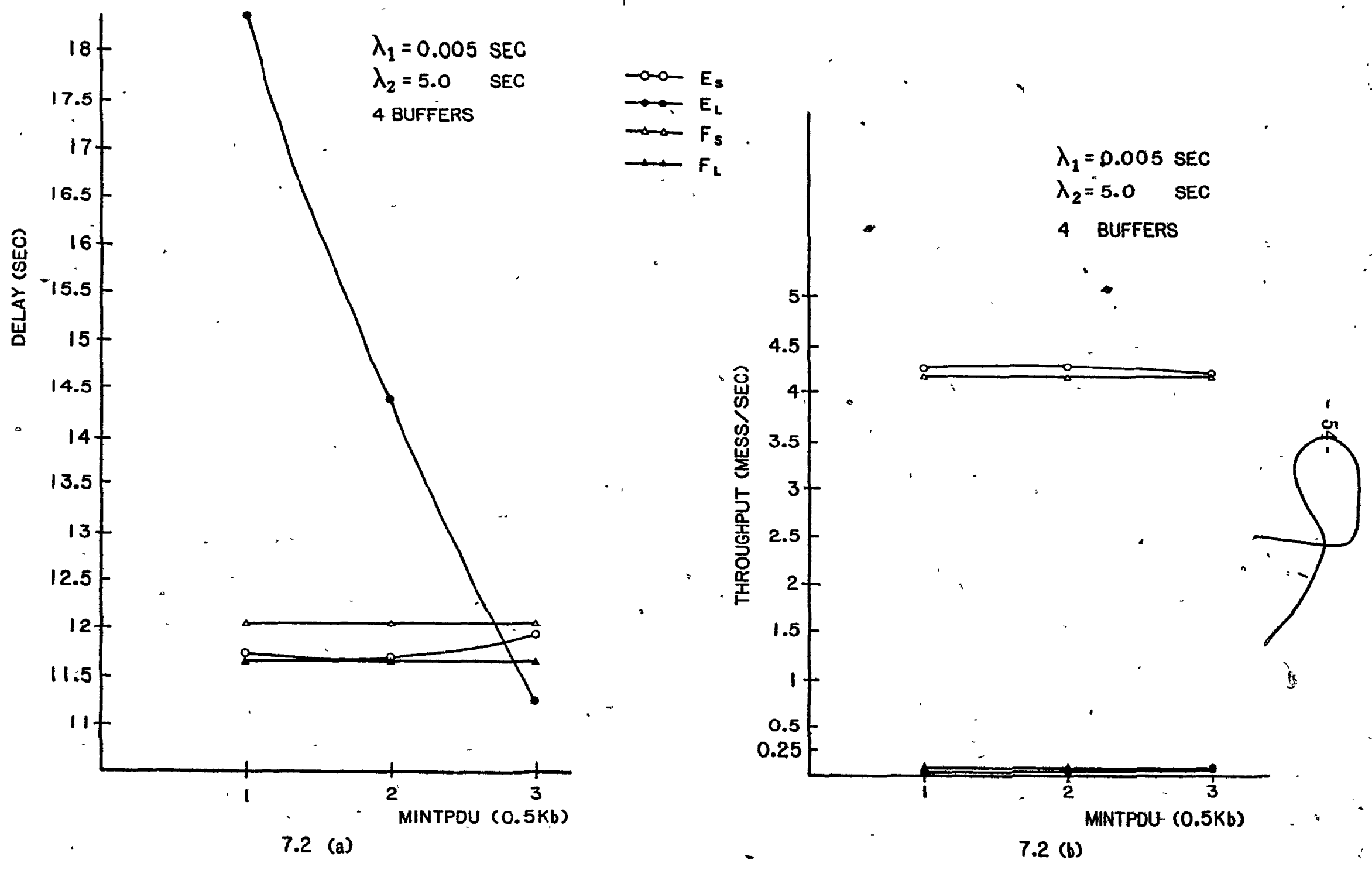

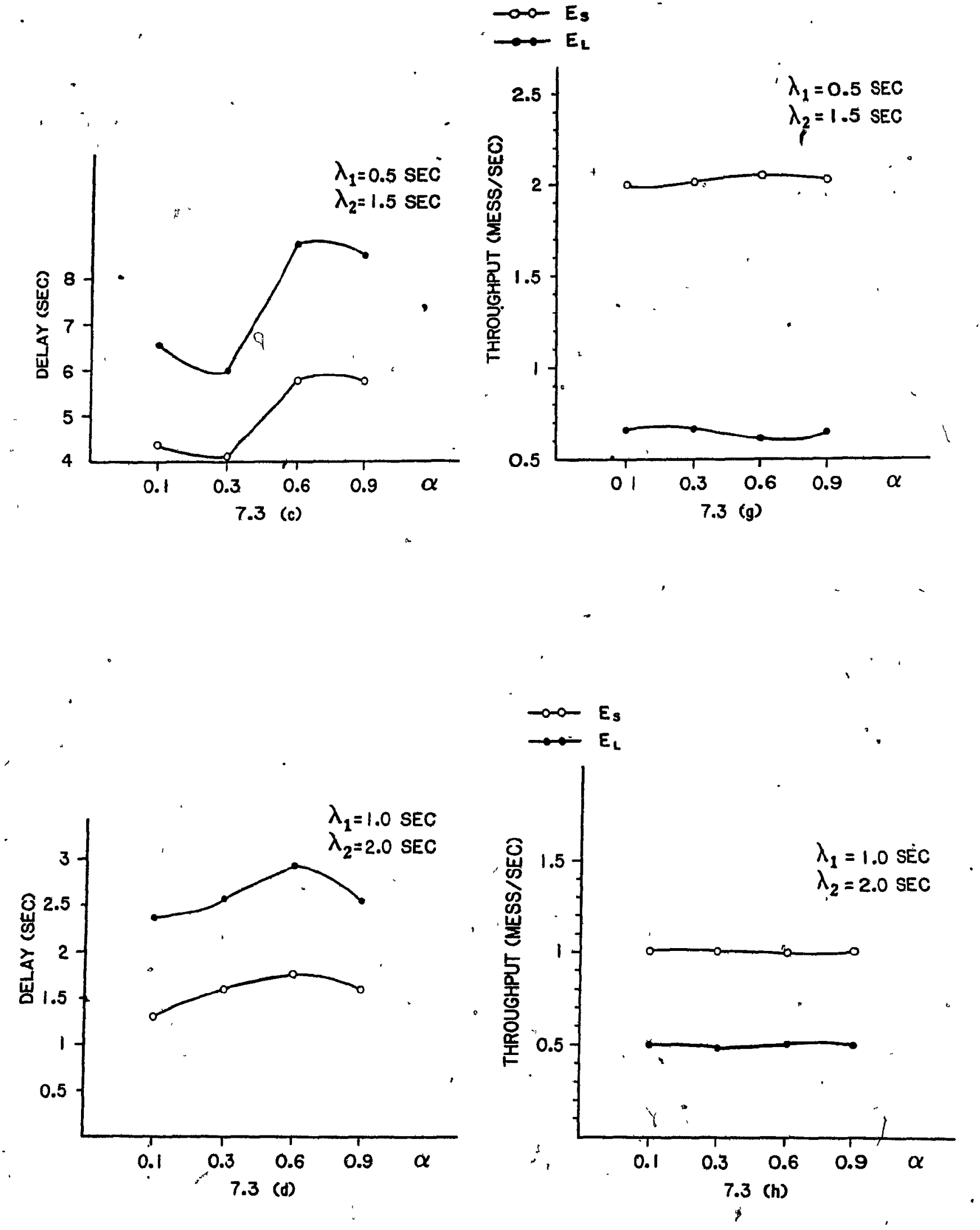
Bibliography

[AUPP83] AUPPERLE, E. Mे., "Merit's Evolution - Statistıcally Speaking", IEEE Trans. on Comp., vol.c-32, no.10, pp. 881-902, Oct. 1983.

[BOX76] BOX, G. E. P. and JENKINS, G. M, Time Series Analysıs, Forecasting and control, revisę edition, Holden Day, Oakland, California, 1976. COHN, S. N., Measurement and Analysis of ARPANET Traffic May 1982 , and March 1983, Bolt Beranek and Newman Inc., May 19833

[EVEQ86] EVEQUOZ, $C$, "On, the Choice of Packet Size in Computer Communication Networks", MSc, School of Compulir Science, McGill University, Montreal, 1986.

[GERL80] GERLA, M, KLEINROCK. L., "Flow Control: A Comparatuve Survey" . IEEEE Trans on Comm, vol COM-28, no 4, pp 553-574, Apr 1980.

[HARB82] HARBITTER, A., TRIPATHI, S, "A Model of Transport level Flow Control", Procee. 1982 SIGMETRICS. Symp. Aug 1982.

[HEYM84] HEYMAN, D $P, S \odot B E L \quad M J$, Stochastic Modols in Operations Research, Volume II Stochastıc Optimization McGraw-Hıll 198.1

[HOWA60] HOWARD, $R$ A, Dynamic Programming and Markov Processes, M.I.T Press, Massachusetts Institute of Technology, Cambridge, Massachusetts, 1960

[HOWA78] IIOWARD, $R A$, "Comments on the Origun and $A$ pplication of Markov Decision Processes", Dynamic Programming And Its Applications, Editor M Puterman, Academic Press Inc., 1979

[KERM80] KERMANI, P., KLEINROCK. L, "Dynamic Flow Control in Storc-andForward Computer Networks", IEEE Trans on Comm., vol.COM-28, 
no.2, pp. 263-271, Feb. 1980.

[KLEI76] KLEINROCK, L., Quéueing Systems Volume II Computer Applications,

Wiley-Interscience, New York, 1976

[ [KLEI80] KLEINROCK. L., KERMANI, P., "Static Flow Control in Store-and-Forward Computer Networks", IEEE Trans on Comm., vol.COM-28, no.2, pp 271-279, Feb 1980

[ISO84á] ISO, International Standard 8072,Information Processing Systems -Open Systems Interconnection- Transport Servace Defintion, International Organization for Standardization,Geneva, 1984.

[NBS83a] . Specification of a Transoport Protocol for Computer Communications, .Volume 1- Overvew ${ }^{\circ}$ and Services, Inst for Computer Sciences and Technology, National Bureau of Standards, Gaithersbụrg, Md., Jan. 1983

[PAWS84] Performance Analyst's Workbench System User's Manual, Information Research Associates, Austın Texas, Nov. 1984

[QUAR86] QUARTERMAN"'J S., HOSKINS, J C, "Nota9le Computer Networks", Comm ACM, vol. 29, no 10, pp 932-971, Oct. 1986

[SCHW87] SCHWARTZ, M, Telecommunications Networks: Protocols, Modeling and Analysis, Addison Wesley, New York, 1987.

[STAL87] ' STALLINGS, 'W., Data and Computer Communications, Macmillan Publishing Company, New York, 1985.

[TANE81] TANENBAUM, A. S., Computer Networks, Prentice Hall, New Jersey, 1981

[TCP83] Transmission Cóntrol Protocol, Military Ständard, directly MIL-STD1778, U.S. Department of Defense, May 20, 1983. 
[ZIMM80] ZIMMERMANN, H., "OSI Reference Model - The ISO Model of Architecture for Open Systems Interconnection", IEEE Tráns. on Comm., vol. COM-28, no.4, pp. 425-432, Apr. 1980.

1
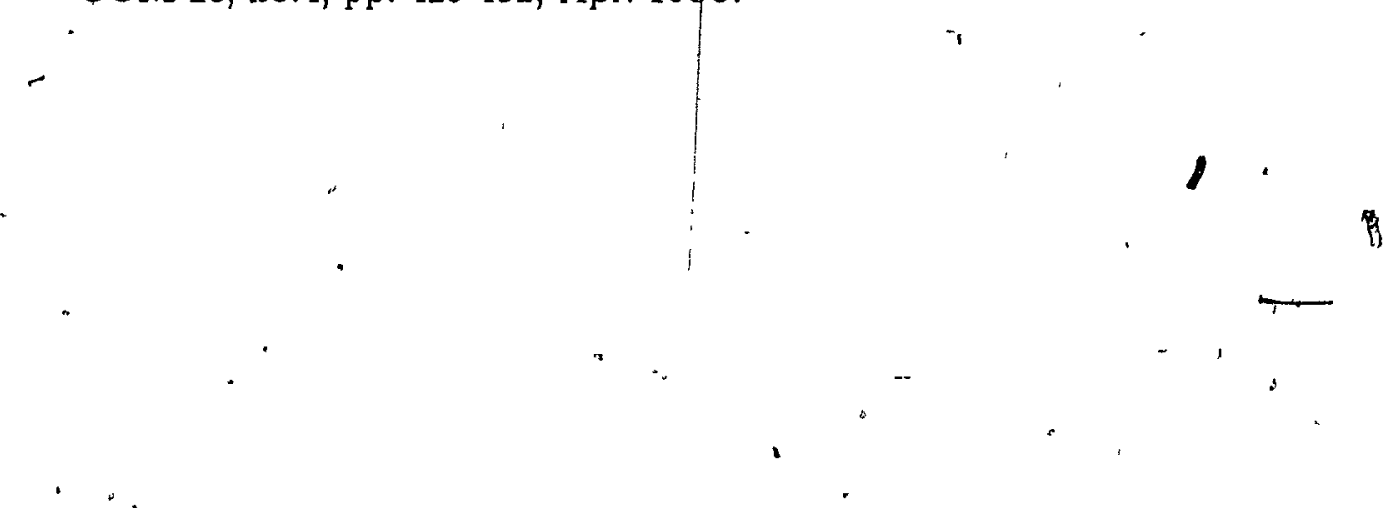

H 